\title{
Hebbian learning and spiking neurons
}

\author{
Richard Kempter* \\ Physik Department, Technische Universität München, D-85747 Garching bei München, Germany
}

Wulfram Gerstner

Swiss Federal Institute of Technology, Center of Neuromimetic Systems, EPFL-DI, CH-1015 Lausanne, Switzerland

J. Leo van Hemmen

Physik Department, Technische Universität München, D-85747 Garching bei München, Germany

(Received 6 August 1998; revised manuscript received 23 November 1998)

\begin{abstract}
A correlation-based ("Hebbian") learning rule at a spike level with millisecond resolution is formulated, mathematically analyzed, and compared with learning in a firing-rate description. The relative timing of presynaptic and postsynaptic spikes influences synaptic weights via an asymmetric "learning window." A differential equation for the learning dynamics is derived under the assumption that the time scales of learning and neuronal spike dynamics can be separated. The differential equation is solved for a Poissonian neuron model with stochastic spike arrival. It is shown that correlations between input and output spikes tend to stabilize structure formation. With an appropriate choice of parameters, learning leads to an intrinsic normalization of the average weight and the output firing rate. Noise generates diffusion-like spreading of synaptic weights. [S1063-651X(99)02804-4]
\end{abstract}

PACS number(s): 87.19.La, 87.19.La, 05.65.+b, 87.18.Sn

\section{INTRODUCTION}

Correlation-based or "Hebbian" learning [1] is thought to be an important mechanism for the tuning of neuronal connections during development and thereafter. It has been shown by various model studies that a learning rule which is driven by the correlations between presynaptic and postsynaptic neurons leads to an evolution of neuronal receptive fields [2-9] and topologically organized maps [10-12].

In all these models, learning is based on the correlation between neuronal firing rates, that is, a continuous variable reflecting the mean activity of a neuron. This is a valid description on a time scale of $100 \mathrm{~ms}$ and more. On a time scale of $1 \mathrm{~ms}$, however, neuronal activity consists of a sequence of short electrical pulses, the so-called action potentials or spikes. During recent years experimental and theoretical evidence has accumulated which suggests that temporal coincidences between spikes on a millisecond or even submillisecond scale play an important role in neuronal information processing [13-24]. If so, a rate description may, and often will, neglect important information that is contained in the temporal structure of a neuronal spike train.

Neurophysiological experiments also suggest that the change of a synaptic efficacy depends on the precise timing of postsynaptic action potentials with respect to presynaptic input spikes on a time scale of $10 \mathrm{~ms}$. Specifically, a synaptic weight is found to increase, if presynaptic firing precedes a postsynaptic spike, and to decrease otherwise [25,26]; see also [27-33]. Our description of learning at a temporal resolution of spikes takes these effects into account.

\footnotetext{
*Electronic address: Richard.Kempter@physik.tu-muenchen.de ${ }^{\dagger}$ Electronic address: Wulfram.Gerstner@di.epfl.ch

‡Electronic address: Leo.van.Hemmen@physik.tu-muenchen.de
}

In contrast to the standard rate models of Hebbian learning, we introduce and analyze a learning rule where synaptic modifications are driven by the temporal correlations between presynaptic and postsynaptic spikes. First steps towards a detailed modeling of temporal relations have been taken for rate models in [34] and for spike models in [22,3543].

\section{DERIVATION OF THE LEARNING EQUATION}

\section{A. Specification of the Hebb rule}

We consider a neuron that receives input from $N \gg 1$ synapses with efficacies $J_{i}, 1 \leqslant i \leqslant N$; cf. Fig. 1 . We assume that changes of $J_{i}$ are induced by presynaptic and postsynaptic spikes. The learning rule consists of three parts. (i) Let $t_{i}^{f}$ be the arrival time of the $f$ th input spike at synapse $i$. The

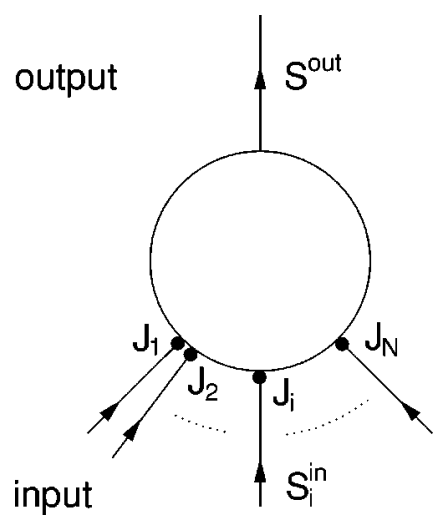

FIG. 1. Single neuron. We study the development of synaptic weights $J_{i}$ (small filled circles, $1 \leqslant i \leqslant N$ ) of a single neuron (large circle). The neuron receives input spike trains denoted by $S_{i}^{\text {in }}$ and produces output spikes denoted by $S^{\text {out }}$. 
arrival of a spike induces the weight $J_{i}$ to change by an amount $\eta w^{\text {in }}$ which can be either positive or negative. The quantity $\eta>0$ is a "small" parameter. (ii) Let $t^{n}$ be the $n$th output spike of the neuron under consideration. This event triggers the change of all $N$ efficacies by an amount $\eta w^{\text {out }}$ which can also be positive or negative. (iii) Finally, time differences between all pairs of input and output spikes influences the change of the efficacies. Given a time difference $s=t_{i}^{f}-t^{n}$ between input and output spikes, $J_{i}$ is changed by an amount $\eta W(s)$ where the learning window $W$ is a realvalued function. It is to be specified shortly; cf. also Fig. 6.

Starting at time $t$ with an efficacy $J_{i}(t)$, the total change $\Delta J_{i}(t)=J_{i}(t+\mathcal{T})-J_{i}(t)$ in a time interval $\mathcal{T}$, which may be interpreted as the length of a learning trial, is calculated by summing the contributions of input and output spikes as well as all pairs of input and output spikes occurring in the time interval $[t, t+\mathcal{T}]$. Denoting the input spike train at synapse $i$ by a series of $\delta$ functions, $S_{i}^{\text {in }}(t)=\Sigma_{f} \delta\left(t-t_{i}^{f}\right)$, and, similarly, output spikes by $S^{\text {out }}(t)=\Sigma_{n} \delta\left(t-t^{n}\right)$, we can formulate the rules (i)-(iii) explicitly by setting

$$
\begin{aligned}
\Delta J_{i}(t)= & \eta \int_{t}^{t+\mathcal{T}} d t^{\prime}\left[w^{\mathrm{in}} S_{i}^{\mathrm{in}}\left(t^{\prime}\right)+w^{\mathrm{out}} S^{\mathrm{out}}\left(t^{\prime}\right)\right] \\
& +\eta \int_{t}^{t+\mathcal{T}} d t^{\prime} \int_{t}^{t+\mathcal{T}} d t^{\prime \prime} W\left(t^{\prime \prime}-t^{\prime}\right) S_{i}^{\mathrm{in}}\left(t^{\prime \prime}\right) S^{\mathrm{out}}\left(t^{\prime}\right) \\
= & \eta\left[\sum_{t_{i}^{f}}^{\prime} w^{\mathrm{in}}+\sum_{t^{n}}^{\prime} w^{\mathrm{out}}+\sum_{t_{i}^{f}, t^{n}}^{\prime} W\left(t_{i}^{f}-t^{n}\right)\right]
\end{aligned}
$$

In Eq. (1b) the prime indicates that only firing times $t_{i}^{f}$ and $t^{n}$ in the time interval $[t, t+\mathcal{T}]$ are to be taken into account; cf. Fig. 2.

Equation (1) represents a Hebb-type learning rule since they correlate presynaptic and postsynaptic behavior. More precisely, here our learning scheme depends on the time sequence of input and output spikes. The parameters $w^{\text {in }}, w^{\text {out }}$ as well as the amplitude of the learning window $W$ may, and in general will, depend on the value of the efficacy $J_{i}$. Such a $J_{i}$ dependence is useful so as to avoid unbounded growth of synaptic weights. Even though we have not emphasized this in our notation, most of the theory developed below is valid for $J_{i}$-dependent parameters; cf. Sec. V B.

\section{B. Ensemble average}

Given that input spiking is random but partially correlated and that the generation of spikes is in general a complicated dynamic process, an analysis of Eq. (1) is a formidable problem. We therefore simplify it. We have introduced a small parameter $\eta>0$ into Eq. (1) with the idea in mind that the learning process is performed on a much slower time scale than the neuronal dynamics. Thus we expect that only averaged quantities enter the learning dynamics.

Considering averaged quantities may also be useful in order to disregard the influence of noise. In Eq. (1) spikes are

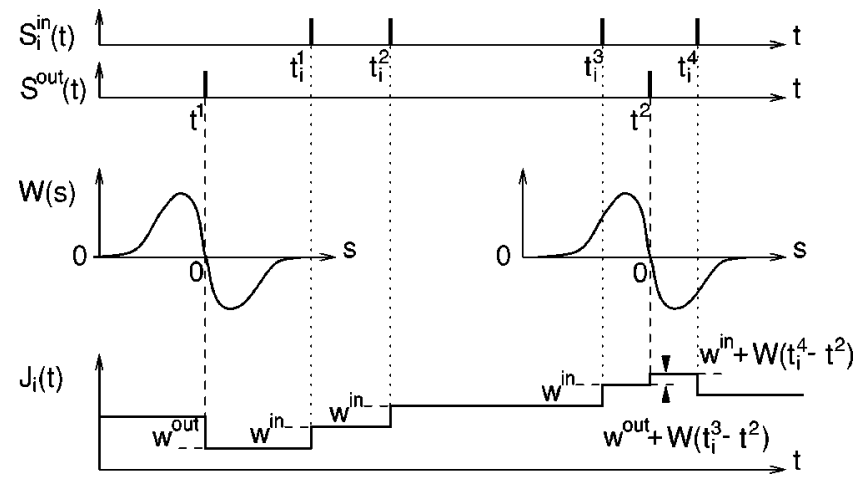

FIG. 2. Hebbian learning and spiking neurons-schematic. In the bottom graph we plot the time course of the synaptic weight $J_{i}(t)$ evoked through input and output spikes (upper graphs, vertical bars). An output spike, e.g., at time $t^{1}$, induces the weight $J_{i}$ to change by an amount $w^{\text {out }}$ which is negative here. To consider the effect of correlations between input and output spikes, we plot the learning window $W(s)$ (center graphs) around each output spike, where $s=0$ matches the output spike times (vertical dashed lines). The three input spikes at times $t_{i}^{1}, t_{i}^{2}$, and $t_{i}^{3}$ (vertical dotted lines) increase $J_{i}$ by an amount $w^{\text {in }}$ each. There is no influence of correlations between these input spikes and the output spike at time $t^{1}$. This becomes visible with the aid of the learning window $W$ centered at $t^{1}$. The input spikes are too far away in time. The next output spike at $t^{2}$, however, is close enough to the previous input spike at $t_{i}^{3}$. The weight $J_{i}$ is changed by $w^{\text {out }}<0$ plus the contribution $W\left(t_{i}^{3}-t^{2}\right)>0$, the sum of which is positive (arrowheads). Similarly, the input spike at time $t_{i}^{4}$ leads to a change $w^{\text {in }}+W\left(t_{i}^{4}\right.$ $\left.-t^{2}\right)<0$.

discrete events that trigger a discontinuous change of the synaptic weight; cf. Fig. 2 (bottom). If we assume a stochastic spike arrival or if we assume a stochastic process for generating output spikes, the change $\Delta J_{i}$ is a random variable, which exhibits fluctuations around some mean drift. Averaging implies that we focus on the drift and calculate the expected rate of change. Fluctuations are treated in Sec. VI.

\section{Self-averaging of learning}

Effective learning needs repetition over many trials of length $\mathcal{T}$, each individual trial being independent of the previous ones. Equation (1) tells us that the results of the individual trials are to be summed. According to the (strong) law of large numbers [44] in conjunction with $\eta$ being "small" [45] we can average the resulting equation, viz., Eq. (1), regardless of the random process. In other words, the learning procedure is self-averaging. Instead of averaging over several trials, we may also consider one single long trial during which input and output characteristics remain constant. Again, if $\eta$ is sufficiently small, time scales are separated and learning is self-averaging.

The corresponding average over the resulting random process is denoted by angular brackets \langle\rangle and is called an ensemble average, in agreement with physical usage. It is a probability measure on a probability space, which need not be specified explicitly. We simply refer to the literature [44]. Substituting $s=t^{\prime \prime}-t^{\prime}$ on the right-hand side of Eq. (1a) and dividing both sides by $\mathcal{T}$, we obtain 


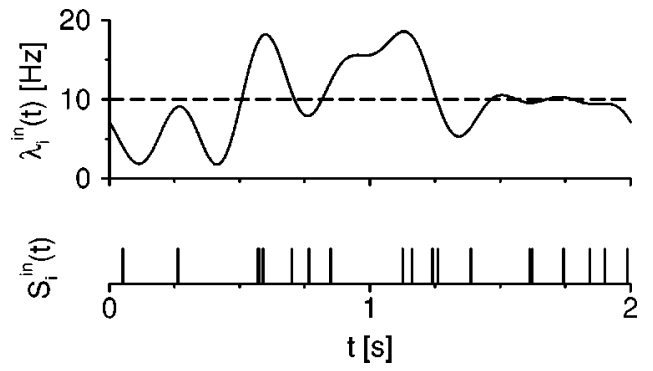

FIG. 3. Inhomogeneous Poisson process. In the upper graph we have plotted an example of an instantaneous rate $\lambda_{i}^{\text {in }}(t)$ in units of $\mathrm{Hz}$. The average rate is $10 \mathrm{~Hz}$ (dashed line). The lower graph shows a spike train $S_{i}^{\text {in }}(t)$ which is a realization of an inhomogeneous Poisson process with rate $\lambda_{i}^{\text {in }}(t)$. The spike times are denoted by vertical bars.

$$
\begin{aligned}
\frac{\left\langle\Delta J_{i}\right\rangle(t)}{\mathcal{T}}= & \frac{\eta}{\mathcal{T}} \int_{t}^{t+\mathcal{T}} d t^{\prime}\left[w^{\text {in }}\left\langle S_{i}^{\text {in }}\right\rangle\left(t^{\prime}\right)+w^{\text {out }}\left\langle S^{\text {out }}\right\rangle\left(t^{\prime}\right)\right] \\
& +\frac{\eta}{\mathcal{T}} \int_{t}^{t+\mathcal{T}} d t^{\prime} \int_{t-t^{\prime}}^{t+\mathcal{T}-t^{\prime}} d s W(s) \\
& \times\left\langle S_{i}^{\text {in }}\left(t^{\prime}+s\right) S^{\text {out }}\left(t^{\prime}\right)\right\rangle .
\end{aligned}
$$

\section{Example: Inhomogeneous Poisson process}

Averaging the learning equation before proceeding is justified if both input and output processes will be taken to be inhomogeneous Poisson processes, which will be assumed throughout Secs. IV-VI. An inhomogeneous Poisson process with time-dependent rate function $\lambda(t) \geqslant 0$ is characterized by two facts: (i) disjoint intervals are independent and (ii) the probability of getting a single event at time $t$ in an interval of length $\Delta t$ is $\lambda(t) \Delta t$, more events having a probability $o(\Delta t)$; see also [46], Appendix A for a simple exposition of the underlying mathematics. The integrals in Eq. (1a) or the sums in Eq. (1b) therefore decompose into many independent events and, thus, the strong law of large numbers applies to them. The output is a temporally local process as well so that the strong law of large numbers also applies to the output spikes at times $t^{n}$ in Eq. (1).

If we describe input spikes by inhomogeneous Poisson processes with intensity $\lambda_{i}^{\text {in }}(t)$, then we may identify the ensemble average over a spike train with the stochastic intensity, $\left\langle S_{i}^{\text {in }}\right\rangle(t)=\lambda_{i}^{\text {in }}(t)$; cf. Fig. 3. The intensity $\lambda_{i}^{\text {in }}(t)$ can be interpreted as the instantaneous rate of spike arrival at synapse $i$. In contrast to temporally averaged mean firing rates, the instantaneous rate may vary on a fast time scale in many biological systems; cf. Sec. III C. The stochastic intensity $\left\langle S^{\text {out }}\right\rangle(t)$ is the instantaneous rate of observing an output spike, where \langle\rangle is an ensemble average over both the input and the output. Finally, the correlation function $\left\langle S_{i}^{\mathrm{in}}\left(t^{\prime \prime}\right) S^{\mathrm{out}}\left(t^{\prime}\right)\right\rangle$ is to be interpreted as the joint probability density for observing an input spike at synapse $i$ at the time $t^{\prime \prime}$ and an output spike at time $t^{\prime}$.

\section{Separation of time scales}

We require the length $\mathcal{T}$ of a learning trial in Eq. (2) to be much larger than typical interspike intervals. Both many input spikes at any synapse and many output spikes should occur on average in a time interval of length $\mathcal{T}$. Then, using the notation $\overline{f(t)}=\mathcal{T}^{-1} \int_{t}^{t+\mathcal{T}} d t^{\prime} f\left(t^{\prime}\right)$, we may introduce the mean firing rates $\nu_{i}^{\text {in }}(t)=\overline{\left\langle S_{i}^{\text {in }}\right\rangle(t)}$ and $\nu^{\text {out }}(t)=\overline{\left\langle S^{\text {out }}\right\rangle(t)}$. We call $\nu_{i}^{\text {in }}$ and $\nu^{\text {out }}$ mean firing rates in order to distinguish them from the previously defined instantaneous rates $\left\langle S_{i}^{\mathrm{in}}\right\rangle$ and $\left\langle S^{\text {out }}\right\rangle$ which are the result of an ensemble average only. Because of their definition, mean firing rates $\nu$ always vary slowly as a function of time. That is, they vary on a time scale of the order of $\mathcal{T}$. The quantities $\nu_{i}^{\text {in }}$ and $\nu^{\text {out }}$ therefore carry hardly any information that may be present in the timing of discrete spikes.

For the sake of further simplification of Eq. (2), we define the width $\mathcal{W}$ of the learning window $W(s)$ and consider the case $\mathcal{T} \gg \mathcal{W}$. Most of the "mass" of the learning window should be inside the interval $[-\mathcal{W}, \mathcal{W}]$. Formally we require $\int_{-\mathcal{W}}^{\mathcal{W}} d s|W(s)| \gg \int_{-\infty}^{-\mathcal{W}} d s|W(s)|+\int_{\mathcal{W}}^{\infty} d s|W(s)|$. For $\mathcal{T} \gg \mathcal{W}$ the integration over $s$ in Eq. (2) can be extended to run from $-\infty$ to $\infty$. With the definition of a temporally averaged correlation function,

$$
C_{i}(s ; t)=\overline{\left\langle S_{i}^{\text {in }}(t+s) S^{\text {out }}(t)\right\rangle},
$$

the last term on the right in Eq. (2) reduces to $\int_{-\infty}^{\infty} d s W(s) C_{i}(s ; t)$. Correlations between presynaptic and postsynaptic spikes, thus, enter spike-based Hebbian learning through $C_{i}$ convolved with the window $W$. We note that the correlation $C_{i}(s ; t)$, though being both an ensemble and a temporally averaged quantity, may change as a function of $s$ on a much faster time scale than $\mathcal{T}$ or the width $\mathcal{W}$ of the learning window. The temporal structure of $C_{i}$ depends essentially on the neuron (model) under consideration. An example is given in Sec. IV A.

We require learning to be a slow process; cf. Sec. II B 1 . More specifically, we require that $J$ values do not change much in the time interval $\mathcal{T}$. Thus $\mathcal{T}$ separates the time scale $\mathcal{W}$ (width of the learning window $W$ ) from the time scale of the learning dynamics, which is proportional to $\eta^{-1}$. Under those conditions we are allowed to approximate the left-hand side of Eq. (2) by the rate of change $d J_{i} / d t$, whereby we have omitted the angular brackets for brevity. Absorbing $\eta$ into the learning parameters $w^{\text {in }}, w^{\text {out }}$, and $W$, we obtain

$$
\frac{d}{d t} J_{i}(t)=w^{\text {in }} \nu_{i}^{\text {in }}(t)+w^{\text {out }} \nu^{\text {out }}(t)+\int_{-\infty}^{\infty} d s W(s) C_{i}(s ; t) .
$$

The ensemble-averaged learning equation (4), which holds for any neuron model, will be the starting point of the arguments below.

\section{SPIKE-BASED AND RATE-BASED HEBBIAN LEARNING}

In this section we indicate the assumptions that are required to reduce spike-based to rate-based Hebbian learning and outline the limitations of the latter.

\section{A. Rate-based Hebbian learning}

In neural network theory, the hypothesis of Hebb [1] is usually formulated as a learning rule where the change of a synaptic efficacy $J_{i}$ depends on the correlation between the 
mean firing rate $\nu_{i}^{\text {in }}$ of the $i$ th presynaptic neuron and the mean firing rate $\nu^{\text {out }}$ of a postsynaptic neuron, viz.,

$$
\begin{aligned}
\frac{d J_{i}}{d t} \equiv & \dot{J}_{i}=a_{0}+a_{1} \nu_{i}^{\text {in }}+a_{2} \nu^{\text {out }} \\
& +a_{3} \nu_{i}^{\text {in }} \nu^{\text {out }}+a_{4}\left(\nu_{i}^{\text {in }}\right)^{2}+a_{5}\left(\nu^{\text {out }}\right)^{2},
\end{aligned}
$$

where $a_{0}<0, a_{1}, a_{2}, a_{3}, a_{4}$, and $a_{5}$ are proportionality constants. Apart from the decay term $a_{0}$ and the "Hebbian", term $\nu_{i}^{\text {in }} \nu^{\text {out }}$ proportional to the product of input and output rates, there are also synaptic changes which are driven separately by the presynaptic and postsynaptic rates. The parameters $a_{0}, \ldots, a_{5}$ may depend on $J_{i}$. Equation (5) is a general formulation up to second order in the rates; see, e.g., $[3,47,12]$.

\section{B. Spike-based Hebbian learning}

To get Eq. (5) from the spike-based learning rule in Eq. (4) two approximations are required. First, if there are no correlations between input and output spikes apart from the correlations contained in the instantaneous rates, we can write $\left\langle S_{i}^{\text {in }}\left(t^{\prime}+s\right) S^{\text {out }}\left(t^{\prime}\right)\right\rangle \approx\left\langle S_{i}^{\text {in }}\right\rangle\left(t^{\prime}+s\right)\left\langle S^{\text {out }}\right\rangle\left(t^{\prime}\right)$. Second, if these rates change slowly as compared to $\mathcal{T}$, then we have $C_{i}(s ; t) \approx \nu_{i}^{\text {in }}(t+s) \nu^{\text {out }}(t)$. In addition, $\nu=\overline{\langle S\rangle}$ is the time evolution on a slow time scale; cf. the discussion after Eq. (3). Since we have $\mathcal{T} \gg \mathcal{W}$, the rates $\nu_{i}^{\text {in }}$ also change slowly as compared to the width $\mathcal{W}$ of the learning window and, thus, we may replace $\nu_{i}^{\text {in }}(t+s)$ by $\nu_{i}^{\text {in }}(t)$ in the correlation term $\int_{-\infty}^{\infty} d s W(s) C_{i}(s ; t)$. This yields $\int_{-\infty}^{\infty} d s W(s) C_{i}(s ; t)$ $\approx \widetilde{W}(0) \nu_{i}^{\text {in }}(t) \nu^{\text {out }}(t)$, where $\widetilde{W}(0):=\int_{-\infty}^{\infty} d s W(s)$. Under the above assumptions we can identify $\widetilde{W}(0)$ with $a_{3}$. By further comparison of Eq. (4) with Eq. (5) we identify $w^{\text {in }}$ with $a_{1}$ and $w^{\text {out }}$ with $a_{2}$, and we are able to reduce Eq. (4) to Eq. (5) by setting $a_{0}=a_{4}=a_{5}=0$.

\section{Limitations of rate-based Hebbian learning}

The assumptions necessary to derive Eq. (5) from Eq. (4), however, are not generally valid. According to the results of Markram et al. [25], the width $\mathcal{W}$ of the Hebbian learning window in cortical pyramidal cells is in the range of $100 \mathrm{~ms}$. At retinotectal synapses $\mathcal{W}$ is also in the range of $100 \mathrm{~ms}$ [26].

A mean rate formulation thus requires that all changes of the activity are slow at a time scale of $100 \mathrm{~ms}$. This is not necessarily the case. The existence of oscillatory activity in the cortex in the range of $40 \mathrm{~Hz}$ (e.g., $[14,15,20,48]$ ) implies activity changes every $25 \mathrm{~ms}$. Retinal ganglion cells fire synchronously at a time scale of about $10 \mathrm{~ms}$ [49]; cf. also [50]. Much faster activity changes are found in the auditory system. In the auditory pathway of, e.g., the barn owl, spikes can be phase-locked to frequencies of up to $8 \mathrm{kHz}$ [51-53]. Furthermore, beyond the correlations between instantaneous rates, additional correlations between spikes may exist.

Because of all the above reasons, the learning rule (5) in the simple rate formulation is insufficient to provide a generally valid description. In Secs. IV and V we will therefore study the full spike-based learning equation (4).

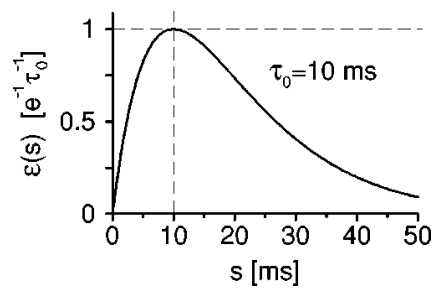

FIG. 4. The postsynaptic potential $\epsilon$ in units of $\left[e^{-1} \tau_{0}^{-1}\right]$ as a function of time $s$ in milliseconds. We have $\epsilon \equiv 0$ for $s<0$ so that $\epsilon$ is causal. The kernel $\epsilon$ has a single maximum at $s=\tau_{0}$. For $s$ $\rightarrow \infty$ the postsynaptic potential $\epsilon$ decays exponentially with time constant $\tau_{0}$; cf. also Appendix B 2 .

\section{STOCHASTICALLY SPIKING NEURONS}

A crucial step in analyzing Eq. (4) is determining the correlations $C_{i}$ between input spikes at synapse $i$ and output spikes. The correlations, of course, depend strongly on the neuron model under consideration. To highlight the main points of learning, we study a simple toy model. Input spikes are generated by an inhomogeneous Poisson process and fed into a stochastically firing neuron model. For this scenario we are able to derive an analytical expression for the correlations between input and output spikes. The introduction of the model and the derivation of the correlation function is the topic of the first subsection. In the second subsection we use the correlation function in the learning equation (4) and analyze the learning dynamics. In the final two subsections the relation to the work of Linsker [3] (a rate formulation of Hebbian learning) and some extensions based on spike coding are considered.

\section{A. Poisson input and stochastic neuron model}

We consider a single neuron which receives input via $N$ synapses $1 \leqslant i \leqslant N$. The input spike trains arriving at the $N$ synapses are statistically independent and generated by an inhomogeneous Poisson process with time-dependent intensities $\left\langle S_{i}^{\text {in }}\right\rangle(t)=\lambda_{i}^{\text {in }}(t)$ with $1 \leqslant i \leqslant N[46]$.

In our simple neuron model we assume that output spikes are generated stochastically with a time-dependent rate $\lambda^{\text {out }}(t)$ that depends on the timing of input spikes. Each input spike arriving at synapse $i$ at time $t_{i}^{f}$ increases (or decreases) the instantaneous firing rate $\lambda^{\text {out }}$ by an amount $J_{i}\left(t_{i}^{f}\right) \epsilon(t$ $-t_{i}^{f}$ ), where $\epsilon$ is a response kernel. The effect of an incoming spike is thus a change in probability density proportional to $J_{i}$. Causality is imposed by the requirement $\epsilon(s)=0$ for $s<0$. In biological terms, the kernel $\epsilon$ may be identified with an excitatory (or inhibitory) postsynaptic potential. In throughout what follows, we assume excitatory couplings $J_{i}>0$ for all $i$ and $\epsilon(s) \geqslant 0$ for all $s$. In addition, the response kernel $\epsilon(s)$ is normalized to $\int d s \epsilon(s)=1$; cf. Fig. 4.

The contributions from all $N$ synapses as measured at the axon hillock are assumed to add up linearly. The result gives rise to a linear inhomogeneous Poisson model with intensity

$$
\lambda^{\text {out }}(t)=\nu_{0}+\sum_{i=1}^{N} \sum_{f} J_{i}\left(t_{i}^{f}\right) \epsilon\left(t-t_{i}^{f}\right) .
$$


Here, $\nu_{0}$ is the spontaneous firing rate and the sums run over all spike arrival times at all synapses. By definition, the spike generation process (6) is independent of previous output spikes. In particular, this Poisson model does not include refractoriness.

In the context of Eq. (4), we are interested in ensemble averages over both the input and the output. Since Eq. (6) is a linear equation, the average can be performed directly and yields

$$
\left\langle S^{\mathrm{out}}\right\rangle(t)=\nu_{0}+\sum_{i=1}^{N} J_{i}(t) \int_{0}^{\infty} d s \epsilon(s) \lambda_{i}^{\mathrm{in}}(t-s) .
$$

In deriving Eq. (7) we have replaced $J_{i}\left(t_{i}^{f}\right)$ by $J_{i}(t)$ because efficacies are assumed to change adiabatically with respect to the width of $\epsilon$. The ensemble-averaged output rate in Eq. (7) depends on the convolution of $\epsilon$ with the input rates. In what follows we denote

$$
\Lambda_{i}^{\mathrm{in}}(t)=\int_{0}^{\infty} d s \epsilon(s) \lambda_{i}^{\mathrm{in}}(t-s) .
$$

Equation (7) may suggest that input and output spikes are statistically independent, which is not the case. To show this explicitly, we determine the ensemble-averaged correlation $\left\langle S_{i}^{\text {in }}(t+s) S^{\text {out }}(t)\right\rangle$ in Eq. (3). Since $\left\langle S_{i}^{\text {in }}(t+s) S^{\text {out }}(t)\right\rangle$ corresponds to a joint probability, it equals the probability density $\lambda_{i}^{\text {in }}(t+s)$ for an input spike at synapse $i$ at time $t+s$ times the conditional probability density of observing an output spike at time $t$ given the above input spike at $t+s$,

$$
\begin{aligned}
& \left\langle S_{i}^{\mathrm{in}}(t+s) S^{\mathrm{out}}(t)\right\rangle \\
& \quad=\lambda_{i}^{\mathrm{in}}(t+s)\left[\nu_{0}+J_{i}(t) \epsilon(-s)+\sum_{j=1}^{N} J_{j}(t) \Lambda_{j}^{\mathrm{in}}(t)\right] .
\end{aligned}
$$

The first term inside the square brackets is the spontaneous output rate and the second term is the specific contribution caused by the input spike at time $t+s$, which vanishes for $s>0$. We are allowed to write $J_{i}(t)$ instead of the "correct" weight $J_{i}(t+s)$; cf. the remark after Eq. (7). To understand the meaning of the second term, we recall that an input spike arriving before an output spike (i.e., $s<0$ ) raises the output firing rate by an amount proportional to $\epsilon(-s)$; cf. Fig. 5 . The sum in Eq. (9) contains the mean contributions of all synapses to an output spike at time $t$. For the proof of Eq. (9), we refer to Appendix A.

Inserting Eq. (9) into Eq. (3) we obtain

$$
\begin{aligned}
C_{i}(s ; t)= & \sum_{j=1}^{N} J_{j}(t) \overline{\lambda_{i}^{\mathrm{in}}(t+s) \Lambda_{j}^{\mathrm{in}}(t)} \\
& +\overline{\lambda_{i}^{\mathrm{in}}(t+s)}\left[\nu_{0}+J_{i}(t) \epsilon(-s)\right],
\end{aligned}
$$

where we have assumed the weights $J_{j}$ to be constant in the time interval $[t, t+\mathcal{T}]$. Temporal averages are denoted by a bar; cf. Sec. II C. Note that $\overline{\lambda_{i}^{\text {in }}(t)}=\nu_{i}^{\text {in }}(t)$.

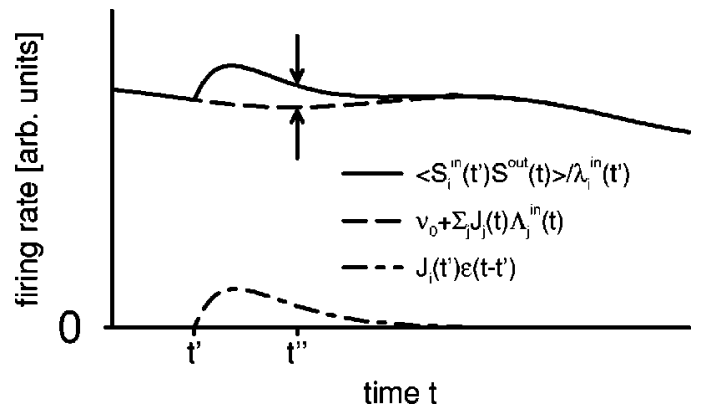

FIG. 5. Spike-spike correlations. To understand the meaning of Eq. (9) we have sketched $\left\langle S_{i}^{\text {in }}\left(t^{\prime}\right) S^{\text {out }}(t)\right\rangle / \lambda_{i}^{\text {in }}\left(t^{\prime}\right)$ as a function of time $t$ (full line). The dot-dashed line at the bottom of the graph is the contribution $J_{i}\left(t^{\prime}\right) \epsilon\left(t-t^{\prime}\right)$ of an input spike occurring at time $t^{\prime}$. Adding this contribution to the mean rate contribution, $\nu_{0}$ $+\sum_{j} J_{j}(t) \Lambda_{j}^{\text {in }}(t)$ (dashed line), we obtain the rate inside the square brackets of Eq. (9) (full line). At time $t^{\prime \prime}>t^{\prime}$ the input spike at time $t^{\prime}$ enhances the output firing rate by an amount $J_{i}\left(t^{\prime}\right) \epsilon\left(t^{\prime \prime}-t^{\prime}\right)$ (arrows). Note that in the main text we have taken $t^{\prime \prime}-t^{\prime}=-s$.

\section{B. Learning equation}

Before inserting the correlation function (10) into the learning rule (4) we define the covariance matrix

$$
q_{i j}(s ; t):=\overline{\left[\lambda_{i}^{\text {in }}(t+s)-\nu_{i}^{\text {in }}(t+s)\right]\left[\Lambda_{j}^{\text {in }}(t)-\nu_{j}^{\text {in }}(t)\right]}
$$

and its convolution with the learning window $W$,

$$
Q_{i j}(t):=\int_{-\infty}^{\infty} d s W(s) q_{i j}(s ; t)
$$

Using Eqs. (7), (10), and (12) in Eq. (4), we obtain

$$
\begin{aligned}
\dot{J}_{i}= & w^{\text {in }} \nu_{i}^{\text {in }}+w^{\text {out }}\left[\nu_{0}+\sum_{j} J_{j} \nu_{j}\right]+\widetilde{W}(0) \nu_{i}^{\text {in }} \nu_{0} \\
& +\sum_{j} J_{j}\left[Q_{i j}+\widetilde{W}(0) \nu_{i}^{\text {in }} \nu_{j}^{\text {in }}+\delta_{i j} \nu_{i}^{\text {in }} \int_{-\infty}^{\infty} d s W(s) \epsilon(-s)\right] .
\end{aligned}
$$

For the sake of brevity, we have omitted the dependence upon time.

The assumption of identical and constant mean input rates, $\nu_{i}^{\text {in }}(t)=\nu^{\text {in }}$ for all $i$, reduces the number of free parameters in Eq. (13) considerably and eliminates all effects coming from rate coding. We define

$$
\begin{gathered}
k_{1}=\left[w^{\text {out }}+\widetilde{W}(0) \nu^{\text {in }}\right] \nu_{0}+w^{\text {in }} \nu^{\text {in }}, \\
k_{2}=\left[w^{\text {out }}+\widetilde{W}(0) \nu^{\text {in }}\right] \nu^{\text {in }}, \\
k_{3}=\nu^{\text {in } \int d s W(s) \epsilon(-s)}
\end{gathered}
$$

in Eq. (13) and arrive at

$$
\dot{J}_{i}=k_{1}+\sum_{j}\left(Q_{i j}+k_{2}+k_{3} \delta_{i j}\right) J_{j} .
$$


Equation (15) describes the ensemble-averaged dynamics of synaptic weights for a spike-based Hebbian learning rule (1) under the assumption of a linear inhomogeneous Poissonian model neuron.

\section{Relation to Linsker's equation}

Linsker [3] has derived a mathematically equivalent equation starting from Eq. (5) and a linear graded-response neuron, a rate-based model. The difference between Linsker's equation and Eq. (15) is, apart from a slightly different notation, the term $k_{3} \delta_{i j}$.

Equation (15) without the $k_{3}$ term has been analyzed extensively by MacKay and Miller [5] in terms of eigenvectors and eigenfunctions of the matrix $Q_{i j}+k_{2}$. In principle, there is no difficulty in incorporating the $k_{3}$ term in their analysis, because $Q_{i j}+k_{2}+\delta_{i j} k_{3}$ contains $k_{3}$ times the unit matrix and thus has the same eigenvectors as $Q_{i j}+k_{2}$. All eigenvalues are simply shifted by $k_{3}$.

The $k_{3}$ term can be neglected if the number $N$ of synapses is large. More specifically, the influence of the $k_{3}$ term as compared to the $k_{2}$ and $Q_{i j}$ term is negligible if for all $i$,

$$
\sum_{j}\left|Q_{i j}+k_{2}\right| J_{j} \gg\left|k_{3}\right| J_{i}
$$

This holds, for instance, if (i) we have many synapses, (ii) $\left|k_{3}\right|$ is smaller than or at most of the same order of magnitude as $\left|k_{2}+Q_{i j}\right|$ for all $i$ and $j$, and (iii) each synapse is weak as compared to the total synaptic weight, $J_{i} \ll \Sigma_{j} J_{j}$. The assumptions (i) -(iii) are often reasonable neurobiological conditions, in particular when the pattern of synaptic weights is still unstructured. The analysis of Eq. (15) presented in Sec. V and focusing on normalization and structure formation is therefore based on these assumptions. In particular, we neglect $k_{3}$.

Nevertheless, our approach even without the $k_{3}$ term is far more comprehensive than Linsker's rate-based ansatz (5) because we have derived Eq. (15) from a spike-based learning rule (1). Therefore correlations between spikes on time scales down to milliseconds or below can enter the driving term $Q_{i j}$ so as to account for structure formation. Correlations on time scales of milliseconds or below may be essential for information processing in neuronal systems; cf. Sec. III C. In contrast to that, Linsker's ansatz is based on a firing-rate description where the term $Q_{i j}$ contains correlations between mean firing rates only. If we use a standard interpretation of rate coding, a mean firing rate corresponds to a temporally averaged quantity which varies on a time scale of the order of hundreds of milliseconds. The temporal structure of spike trains is neglected completely.

Finally, our ansatz (1) allows the analysis of the influence of noise on learning. Learning results from stepwise weight changes. Each weight performs a random walk whose expectation value is described by the ensemble-averaged equation (15). Analysis of noise as a deviation from the mean is deferred to Sec. VI.

\section{Stabilization of learning}

We now discuss the influence of the $k_{3}$ term in Eq. (15). It gives rise to an exponential growth or decay of weights,

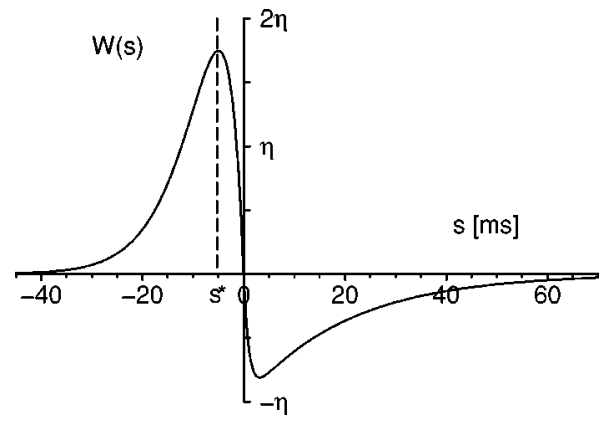

FIG. 6. The learning window $W$ in units of the learning parameter $\eta$ as a function of the delay $s=t_{i}^{f}-t^{n}$ between presynaptic spike arrival at synapse $i$ at time $t_{i}^{f}$ and postsynaptic firing at time $t^{n}$. If $W(s)$ is positive (negative) for some $s$, the synaptic efficacy $J_{i}$ is increased (decreased). The increase of $J_{i}$ is most efficient if a presynaptic spike arrives a few milliseconds before the postsynaptic neuron starts firing (vertical dashed line at $s=s^{*}$ ). For $|s| \rightarrow \infty$ we have $W(s) \rightarrow 0$. The form of the learning window and parameter values are as described in Appendix B 1.

depending on the sign of $k_{3}$. Since firing rates $\nu$ are always positive and $k_{3}=\nu^{\text {in }} \int d s W(s) \epsilon(-s)$, the sign of the integral $\int d s W(s) \epsilon(-s)$ is crucial. Hebb's principle suggests that for excitatory synapses, the integral is always positive. To understand why, let us recall that $s$ is defined as the time difference between input and output spikes. The response kernel $\epsilon$ vanishes for negative arguments. Thus the integral effectively runs only over negative $s$. According to our definition, $s<0$ implies that presynaptic spikes precede postsynaptic firing. These are the spikes that may have participated in firing the postsynaptic neuron. Hebb's principle [1] suggests that these synapses are strengthened, hence $W(s)>0$ for $s<0$; cf. Fig. 6. This idea is also in agreement with recent neurobiological results $[25,26,33]$ : Only those synapses are potentiated where presynaptic spikes arrive a few milliseconds before a postsynaptic spike occurs so that the former arrive "in time." We conclude that $\int d s W(s) \epsilon(-s)$ $>0$ and, hence, the $k_{3}$ term is positive.

With $k_{3}>0$ every weight and thus every structure in the distribution of weights is enhanced. This may contribute to the stability of structured weight distributions at the end of learning, in particular when the synapses are few and strong $[22,54]$. In this case, Eq. (16) may be not fulfilled and the $k_{3}$ term in Eq. (15) has an important influence. Thus spikebased learning is different from simple rate-based learning rules. Spike-spike correlations on a millisecond time scale play an important role and tend to stabilize existing strong synapses.

\section{LEARNING DYNAMICS}

In order to get a better understanding of the principal features of the learning dynamics, we discuss Eq. (15) with $k_{3}=0$ for a particularly simple configuration: a model with two groups of synapses. Input rates are homogeneous within each group but different between one group and the other. Our discussion focuses on intrinsic normalization of output rates and structure formation. We take lower and upper bounds for the $J$ values into account explicitly and consider the limiting case of weak correlations in the input. We will see that for a realistic scenario we need to require $w^{\text {in }}>0$ and 
TABLE I. Values of the parameters used for the numerical simulations (a) and derived quantities (b).

(a) Parameters

\begin{tabular}{|c|c|}
\hline Learning & $\begin{aligned} \eta & =10^{-5} \\
w^{\text {in }} & =\eta \\
w^{\text {out }} & =-1.0475 \eta \\
A_{+} & =1 \\
A_{-} & =-1 \\
\tau_{+} & =1 \mathrm{~ms} \\
\tau_{-} & =20 \mathrm{~ms} \\
\tau^{\text {syn }} & =5 \mathrm{~ms}\end{aligned}$ \\
\hline $\begin{array}{l}\text { EPSP } \\
\text { Synaptic input }\end{array}$ & $\begin{aligned} \tau_{0} & =10 \mathrm{~ms} \\
N & =50 \\
M_{1} & =25 \\
M_{2} & =25 \\
\nu^{\text {in }} & =10 \mathrm{~s}^{-1} \\
\delta \nu^{\text {in }} & =10 \mathrm{~s}^{-1} \\
\omega /(2 \pi) & =40 \mathrm{~s}^{-1}\end{aligned}$ \\
\hline Further parameters & $\begin{aligned} \nu_{0} & =0 \\
\vartheta & =0.1 \\
\text { (b) Derived quantities } & \\
\widetilde{W}(0):=\int d s W(s) & =4.75 \times 10^{-8} \mathrm{~s} \\
\int d s W(s)^{2} & =3.68 \times 10^{-12} \mathrm{~s} \\
\int d s W(s) \epsilon(-s) & =7.04 \times 10^{-6} \\
Q & =6.84 \times 10^{-7} \mathrm{~s}^{-1} \\
k_{1} & =1 \times 10^{-4} \mathrm{~s}^{-1} \\
k_{2} & =-1 \times 10^{-4} \mathrm{~s}^{-1} \\
k_{3} & =7.04 \times 10^{-5} \mathrm{~s}^{-1} \\
\tau^{\mathrm{av}} & =2 \times 10^{2} \mathrm{~s} \\
\tau^{\mathrm{str}} & =2.93 \times 10^{4} \mathrm{~s}^{\mathrm{noise}} \\
\tau^{\mathrm{nois}} & =1.62 \times 10^{5} \mathrm{~s}^{\mathrm{av}} \\
J_{*} & =2 \times 10^{-2} \\
D & =2.47 \times 10^{-9} \mathrm{~s}^{-1} \\
D^{\prime} & =1.47 \times 10^{-9} \mathrm{~s}^{-1}\end{aligned}$ \\
\hline
\end{tabular}

$w^{\text {out }}<0$ and that we can formulate theoretical predictions of the relative magnitude of the learning parameters $w^{\text {in }}, w^{\text {out }}$ and the form of the learning window $W$. The theoretical considerations are illustrated by numerical simulations whose parameters are justified in Appendix B and summarized in Table I.

\section{A. Models of synaptic input}

We divide the $N$ statistically independent synapses, all converging onto the very same neuron, into two groups, $\mathcal{M}_{1}$ and $\mathcal{M}_{2}$. The numbers of synapses are $M_{1}$ and $M_{2}$, respectively, where $M_{1}+M_{2}=N$ and $M_{1}, M_{2} \gg 1$. Since each group contains many synapses, we may assume that $M_{1}$ and $M_{2}$ are of the same order of magnitude. The spike input at synapses $i$ in group $\mathcal{M}_{1}$ is generated by a Poisson process with a constant intensity $\lambda_{i}^{\text {in }}(t) \equiv \nu^{\text {in }}$, which is independent of $t$. We therefore have $Q_{i j}(t) \equiv 0$ for $i$ or $j \in \mathcal{M}_{1}$; cf. Eqs. (11) and (12). The synapses $i$ in group $\mathcal{M}_{2}$ are driven by an arbitrary time-dependent input, $\lambda_{i}^{\text {in }}(t)=\lambda^{\text {in }}(t)$, with the same mean input rate $\nu^{\text {in }}=\overline{\lambda^{\text {in }}(t)}$ as in group $\mathcal{M}_{1}$. Without going into details about the dependence of $\lambda^{\text {in }}(t)$ upon the time $t$, we assume $\lambda^{\text {in }}(t)$ to be such that the covariance $q_{i j}(s ; t)$ in Eq. (11) is independent of $t$. In this case it follows from Eq. (12) that $Q_{i j}(t) \equiv Q$ for $i, j \in \mathcal{M}_{2}$, regardless of $t$. For the sake of simplicity we require in addition that $Q>0$. In summary, we suppose in the following

$$
Q_{i j}(t)= \begin{cases}Q>0 & \text { for } i, j \in \mathcal{M}_{2}, \\ 0 & \text { otherwise }\end{cases}
$$

We recall that $Q_{i j}$ is a measure of the correlations in the input arriving at synapses $i$ and $j$; cf. Eqs. (11) and (12). Equation (17) states that at least some of the synapses receive positively correlated input, a rather natural assumption. Three different realizations of Eq. (17) are now discussed in turn.

\section{White-noise input}

For all synapses in group $\mathcal{M}_{2}$, let us consider the case of stochastic white-noise input with intensity $\lambda^{\text {in }}(t)$ and mean firing rate $\overline{\lambda^{\mathrm{in}}(t)}=\nu^{\mathrm{in}}(t) \geqslant 0$. The fluctuations are $\overline{\left[\lambda^{\text {in }}(t+s)-\nu^{\text {in }}(t+s)\right]\left[\lambda^{\text {in }}(t)-\nu^{\text {in }}(t)\right]}=\sigma_{0} \delta(s)$. Due to the convolution (8) with $\epsilon$, Eq. (11) yields $q_{i j}(s ; t)=\sigma_{0} \epsilon(-s)$, independently of $t, i$, and $j$. We use Eq. (12) and find $Q_{i j}(t) \equiv Q=\sigma_{0} \int d s W(s) \epsilon(-s)$. We want $Q>0$ and therefore arrive at $\int W(s) \epsilon(-s)=k_{3} / \nu^{\text {in }}>0$. We have seen before in Sec. IV D that $k_{3}>0$ is a natural assumption and in agreement with experiments.

\section{Colored-noise input}

Let us now consider the case of an instantaneous and memoryless excitation, $\epsilon(s)=\delta(s)$. We assume that $\lambda^{\text {in }}$ $-\nu^{\text {in }}$ obeys a stationary Ornstein-Uhlenbeck process [62] with correlation time $\tau_{c}$. The fluctuations are therefore $q_{i j}(s ; t) \propto \exp \left(-|s| / \tau_{c}\right)$, independent of the synaptic indices $i$ and $j . Q>0$ implies $\int d s W(s) \exp \left(-|s| / \tau_{c}\right)>0$.

\section{Periodic input}

Motivated by oscillatory neuronal activity in the auditory system and in the cortex (cf. Sec. III C), we now consider the scenario of periodically modulated rates $\left[\lambda^{\text {in }}(t)-\nu^{\text {in }}\right]$ $=\delta \nu^{\text {in }} \cos (\omega t)$, where $\omega \gg 2 \pi / \mathcal{T}$. Let us first study the case $\epsilon(s)=\delta(s)$. We find $Q=\left(\delta \nu^{\text {in }}\right)^{2} / 2 \int d s W(s) \cos (\omega s)$. Positive $Q$ hence requires the real part of the Fourier transform $\widetilde{W}(\omega):=\int d s W(s) \exp (i \omega s)$ to be positive, i.e., $\operatorname{Re}[\widetilde{W}(\omega)]$ $>0$. For a general interaction kernel $\epsilon(s)$, we find $q_{i j}(s ; t)$ $=\left(\delta \nu^{\text {in }}\right)^{2} / 2 \int d s^{\prime} \epsilon\left(s^{\prime}\right) \cos \left[\omega\left(s+s^{\prime}\right)\right]$ and hence

$$
Q=\left(\delta \nu^{\text {in }}\right)^{2} / 2 \operatorname{Re}[\tilde{W}(\omega) \tilde{\epsilon}(\omega)]
$$

independent of $t$. Then $Q>0$ requires $\operatorname{Re}[\widetilde{W}(\omega) \tilde{\epsilon}(\omega)]>0$.

\section{B. Normalization}

Normalization is a very desirable property for any learning rule. It is a natural requirement that the average weight and the mean output rate do not blow up during learning but 

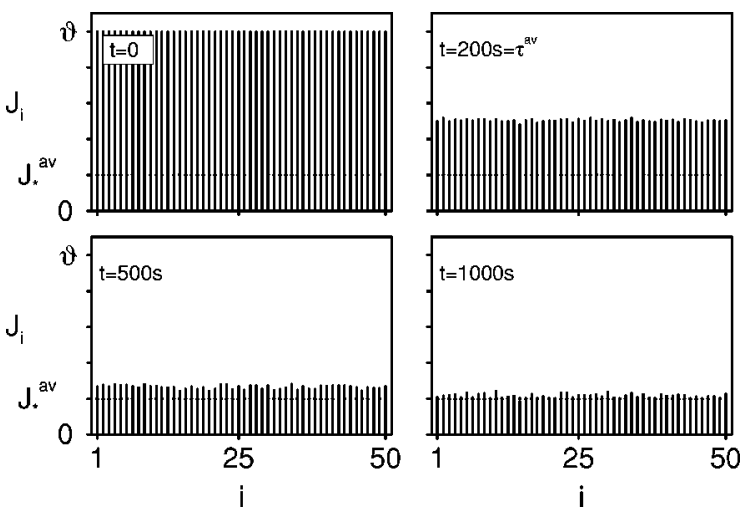

FIG. 7. Numerical simulation of weight normalization with parameters as given in Appendix B. The four graphs show the temporal evolution of synaptic weights $J_{i}, 1 \leqslant i \leqslant 50$, before $(t=0)$ and during learning ( $t=200,500$, and $1000 \mathrm{~s})$. Before learning, all weights are initialized at the upper bound $\vartheta=0.1$. During learning, weights decrease towards the fixed point of the average weight, $J_{*}^{\text {av }}=2.0 \times 10^{-2}$; cf. also Fig. 8, topmost full line. The time constant of normalization is $\tau^{\mathrm{av}}=2.0 \times 10^{2} \mathrm{~s}$, which is much smaller than the time constant of structure formation; cf. Sec. V C and Fig. 9. For times $t \leqslant 1000 \mathrm{~s}$ we therefore can neglect effects coming from structure formation.

are stabilized at a reasonable value in an acceptable amount of time. Standard rate-based Hebbian learning can lead to unlimited growth of the average weight. Several methods have been designed to control this unlimited growth; for instance, subtractive or multiplicative rescaling of the weights after each learning step so as to impose either $\Sigma_{j} J_{j}=$ const or else $\Sigma_{j} J_{j}^{2}=$ const; cf., e.g., $[2,7,55]$. It is hard to see, however, where this should come from. Furthermore, a $J$ dependence of the parameters $a_{1}, \ldots, a_{5}$ in the learning equation (5) is often assumed. Higher-order terms in the expansion (5) may also be used to control unlimited growth.

In this subsection we show that under some mild conditions there is no need whatsoever to invoke the $J$ dependence of the learning parameters, rescaling of weights, or higherorder correlations to get normalization, which means here that the average weight

$$
J^{\mathrm{av}}=\frac{1}{N} \sum_{i=1}^{N} J_{i}
$$

approaches a stable fixed point during learning. Moreover, in this case the mean output rate $\nu^{\text {out }}$ is also stabilized since $\nu^{\text {out }}=\nu_{0}+N J^{\text {av }} \nu^{\text {in }}$; cf. Eq. (7).

As long as the learning parameters do not depend on the $J$ values, the rate of change of the average weight is obtained from Eqs. (15), (19), and $k_{3}=0$ (Sec. IV C),

$$
\dot{J}^{\mathrm{av}}=k_{1}+N k_{2} J^{\mathrm{av}}+N^{-1} \sum_{i, j} Q_{i j} J_{j} .
$$

In the following we consider the situation at the beginning of the learning procedure where the set of weights $\left\{J_{i}\right\}$ has not picked up any correlations with the set of Poisson intensities $\left\{\lambda_{i}^{\text {in }}\right\}$ yet and therefore is independent. We may then replace $J_{i}$ and $Q_{i j}$ on the right-hand side of Eq. (20) by their average values $J^{\text {av }}$ and $Q^{\text {av }}=N^{-2} \Sigma_{i, j}^{N} Q_{i j}$, respectively. The

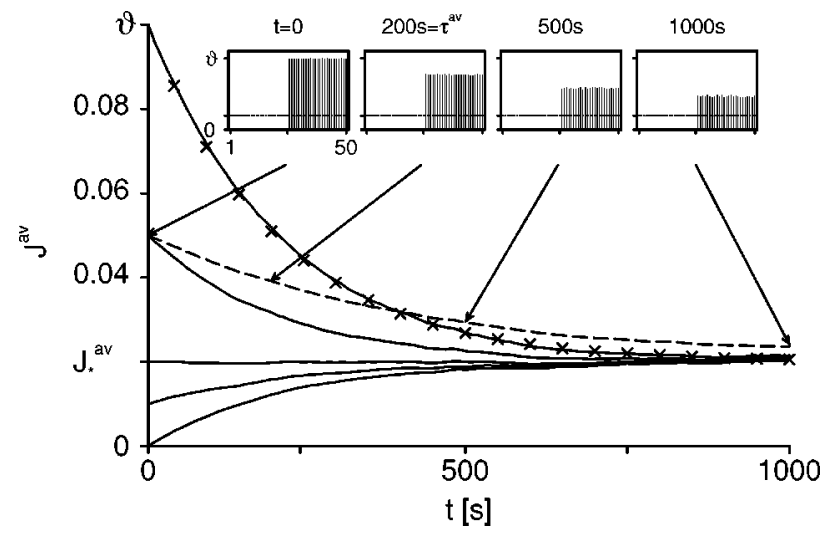

FIG. 8. Development of the average weight $J^{\text {av }}$ as a function of time $t$ in units of $10^{3} \mathrm{~s}$. The simulations we started at $t=0$ with five different average weights, $J^{\text {av }} \in\left\{0,0.01,0.02=J_{*}^{\text {av }}, 0.05,0.1\right.$ $=\vartheta\}$. Full lines indicate homogeneous initial weight distributions, where $J_{i}=J^{\text {av }}$ at $t=0$ for all $i$; cf. also Fig. 7, upper left panel. In all five cases, $J^{\text {av }}$ decays with the time constant $\tau^{\mathrm{av}}=2.0 \times 10^{2} \mathrm{~s}$ describing the rate of normalization to the fixed point $J_{*}^{\mathrm{av}}=2.0$ $\times 10^{-2}$. Our theoretical prediction according to Sec. V B (crosses on the uppermost full line) is in good agreement with the numerical results. The dashed line indicates the development of $J^{\text {av }}$ starting from an inhomogeneous initial weight distribution $J_{i}=0$ for $1 \leqslant i$ $\leqslant 25$ and $J_{i}=\vartheta$ for $25<i \leqslant 50=N$. In the inhomogeneous case, $\tau^{\text {av }}$ is enlarged as compared to the homogeneous case by a factor of 2 because only half of the synapses are able to contribute to normalization; cf. Appendix D. The insets (signatures as in Fig. 7) show the inhomogeneous weight distributions (arrows) at times $t$ $=0,200,500$, and $1000 \mathrm{~s}$; the dotted line indicates the fixed point $J_{*}^{\mathrm{av}}=0.2 \vartheta$. We note that here the distribution remains inhomogeneous.

specific input (17) described in the preceding section yields $Q^{\text {av }}=\left(M_{2} / N\right)^{2} Q>0$. We rewrite Eq. (20) in the standard form $\dot{J}^{\text {av }}=\left[J_{*}^{\text {av }}-J^{\text {av }}\right] / \tau^{\text {av }}$, where

$$
J_{*}^{\mathrm{av}}=-k_{1} /\left[N\left(k_{2}+Q^{\mathrm{av}}\right)\right]
$$

is the fixed point for the average weight and

$$
\tau^{\mathrm{av}}=J_{*}^{\mathrm{av}} / k_{1}=-1 /\left[N\left(k_{2}+Q^{\mathrm{av}}\right)\right]
$$

is the time constant of normalization. The fixed point in Eq. (21) is stable, if and only if $\tau^{\mathrm{av}}>0$.

During learning, weights $\left\{J_{i}\right\}$ and rates $\left\{\lambda_{i}^{\text {in }}\right\}$ may become correlated. In Appendix $\mathrm{C}$ we demonstrate that the influence of any interdependence between weights and rates on normalization can be neglected in the case of weak correlations in the input,

$$
0<Q \ll-k_{2} \text {. }
$$

The fixed point $J_{*}^{\mathrm{av}}$ in Eq. (21) and the time constant $\tau^{\mathrm{av}}$ in Eq. (22) are, then, almost independent of the average correlation $Q^{\text {av }}$, which is always of the same order as $Q$.

In Figs. 7 and 8 we show numerical simulations with parameters as given in Appendix B. The average weight $J^{\text {av }}$ always approaches $J_{*}^{\text {av }}$, independent of any initial conditions in the distribution of weights. 


\section{Weight constraints lead to further conditions on learning} parameters

We have seen that normalization is possible without a $J$ dependence of the learning parameters. Even if the average weight $J^{\text {av }}$ approaches a fixed point $J_{*}^{\text {av }}$, there is no restriction for the size of individual weights, apart from $J_{i} \geqslant 0$ for excitatory synapses and $J_{i} \lesssim N J_{*}^{\text {av }}$. This means that a single weight at most comprises the total (normalized) weight of all $N$ synapses. The latter case is, however, unphysiological, since almost every neuron holds many synapses with nonvanishing efficacies (weights) and efficacies of biological synapses seem to be limited. We take this into account in our learning rule by introducing a hard upper bound $\vartheta$ for each individual weight. As we will demonstrate, a reasonable value of $\vartheta$ does not influence normalization in that $J_{*}^{\text {av }}$ remains unchanged. However, an upper bound $\vartheta>0$, whatever its value, leads to further constraints on the learning parameters.

To incorporate the restricted range of individual weights into our learning rule (1), we assume that we can treat the learning parameters $w^{\text {in }}, w^{\text {out }}$, and the amplitude of $W$ to be constant in the range $0 \leqslant J_{i} \leqslant \vartheta$. For $J_{i}<0$ or $J_{i}>\vartheta$, we take $w^{\text {in }}=w^{\text {out }}=W=0$. In other words, we use Eq. (15) only between the lower bound 0 and the upper bound $\vartheta$ and set $d J_{i} / d t=0$ if $J_{i}<0$ or $J_{i}>\vartheta$.

Because of lower and upper bounds for each synaptic weight, $0 \leqslant J_{i} \leqslant \vartheta$ for all $i$, a realizable fixed point $J_{*}^{\text {av }}$ has to be within these limits. Otherwise all weights saturate either at the lower or at the upper bound. To avoid this, we first of all need $J_{*}^{\mathrm{av}}>0$. Since $\tau^{\mathrm{av}}=J_{*}^{\mathrm{av}} / k_{1}$ in Eq. (22) must be positive for stable fixed points, we also need $k_{1}>0$. The meaning becomes transparent from Eq. (14) in the case of vanishing spontaneous activity in the output, $\nu_{0}=0$. Then $k_{1}>0$ reduces to

$$
w^{\text {in }}>0 \text {, }
$$

which corresponds to neurobiological reality [28,56,31].

A second condition for a realizable fixed point arises from the upper bound $\vartheta>J_{*}^{\text {av }}$. This requirement leads to $k_{2}<-k_{1} /(N \vartheta)-Q^{\text {av }}$. Exploiting only $k_{2}<0$, we find from Eq. (14) that $w^{\text {out }}+\widetilde{W}(0) \nu^{\text {in }}<0$, which means that postsynaptic spikes on average reduce the total weight of synapses. This is one of our predictions that can be tested experimentally. Assuming $\widetilde{W}(0)>0$, which seems reasonable - with the benefit of hindsight - in terms of rate-coded learning in the manner of Hebb (Sec. III), we predict

$$
w^{\text {out }}<0
$$

which has not been verified by experiments yet.

Weight constraints do not influence the position of the fixed point $J_{*}^{\text {av }}$ (as long as it remains realizable) but may enlarge the value of the time constant $\tau^{\text {av }}$ of normalization (see details in Appendix D). The time constant $\tau^{\mathrm{av}}$ changes because weights saturated at the lower (upper) bound cannot contribute to a decrease (increase) of $J^{\text {av }}$. If fewer than the total number of weights add to our (subtractive) normalization, then the fixed point is approached more slowly; cf. Fig. 8 , dashed line and insets. The factor, however, by which $\tau^{\text {av }}$ may be enlarged is of order 1 , if we take the upper bound to be $\vartheta=(1+d) J_{*}^{\text {av }}$, where $d>0$ is of order 1 , which will be assumed throughout what follows; cf. Appendix D.

\section{Structure formation}

In our simple model with two groups of input, structure formation can be measured by the difference $J^{\text {str }}$ between the average synaptic strength in groups $\mathcal{M}_{1}$ and $\mathcal{M}_{2}$; cf. Sec. V A. We derive conditions under which this difference increases during learning. In the course of the argument we also show that structure formation takes place on a time scale $\tau^{\text {str }}$ considerably slower than the time scale $\tau^{\text {av }}$ of normalization.

We start from Eq. (15) with $k_{3}=0$ and randomly distributed weights. For the moment we assume that normalization has already taken place. Furthermore, we assume small correlations as in Eq. (23), which assures that the fixed point $J_{*}^{\text {av }} \approx-k_{1} /\left(N k_{2}\right)$ is almost constant during learning; cf. Eqs. (21) and (C1). If the formation of any structure in $\left\{J_{i}\right\}$ is slow as compared to normalization, we are allowed to use $J^{\text {av }}=J_{*}^{\text {av }}$ during learning. The consistency of this ansatz is checked at the end of this section.

The average weight in each of the two groups $\mathcal{M}_{1}$ and $\mathcal{M}_{2}$ is

$$
J^{(1)}=\frac{1}{M_{1}} \sum_{i \in \mathcal{M}_{1}} J_{i} \quad \text { and } \quad J^{(2)}=\frac{1}{M_{2}} \sum_{i \in \mathcal{M}_{2}} J_{i} .
$$

If lower and upper bounds do not influence the dynamics of each weight, the corresponding rates of change are

$$
\begin{gathered}
\dot{J}^{(1)}=k_{1}+M_{1} J^{(1)} k_{2}+M_{2} J^{(2)} k_{2}, \\
\dot{J}^{(2)}=k_{1}+M_{2} J^{(2)}\left(k_{2}+Q\right)+M_{1} J^{(1)} k_{2} .
\end{gathered}
$$

One expects the difference $J^{\text {str }}=J^{(2)}-J^{(1)}$ between those average weights to grow during learning because group $\mathcal{M}_{2}$ receives a stronger reinforcement than $\mathcal{M}_{1}$. Differentiating $J^{\text {str }}$ with respect to time, using Eq. (27) and the constraint $J^{\mathrm{av}}=J_{*}^{\mathrm{av}}=N^{-1}\left(M_{1} J^{(1)}+M_{2} J^{(2)}\right)$, we find the rate of growth

$$
\dot{J}^{\mathrm{str}}=\frac{M_{1} M_{2}}{N} Q J^{\mathrm{str}}+M_{2} Q J_{*}^{\mathrm{av}} .
$$

The first term on the right-hand side gives rise to an exponential increase $(Q>0)$ while the second term gives rise to a linear growth of $J^{\text {str }}$. Equation (28) has an unstable fixed point at $J_{*}^{\text {str }}=-N / M_{1} J_{*}^{\text {av }}$. Note that $J_{*}^{\text {str }}$ is always negative and independent of $Q$.

We associate the time constant $\tau^{\mathrm{str}}$ of structure formation with the time that is necessary for an increase of $J^{\text {str }}$ from a typical initial value to its maximum. The maximum of $J^{\text {str }}$ is of order $J_{*}^{\text {av }}$ if $M_{1} / M_{2}$ is of order 1 (Sec. V A) and if $\vartheta$ $=(1+d) J_{*}^{\mathrm{av}}$, where $d>0$ is of order 1 (Sec. V B). At the beginning of learning $(t=0)$ we may take $J^{\mathrm{str}}(0)=0$. Using this initial condition, an integration of Eq. (28) leads to $J^{\mathrm{str}}(t)=\left(N / M_{1}\right) J_{*}^{\mathrm{av}}\left[\exp \left(t M_{1} M_{2} Q / N\right)-1\right]$. With $t=\tau^{\text {str }}$ and $J^{\mathrm{str}}\left(\tau^{\mathrm{str}}\right)=J_{*}^{\text {av }}$ we obtain $\tau^{\mathrm{str}}=N /\left(M_{1} M_{2} Q\right) \ln \left(M_{1} / N+1\right)$. 


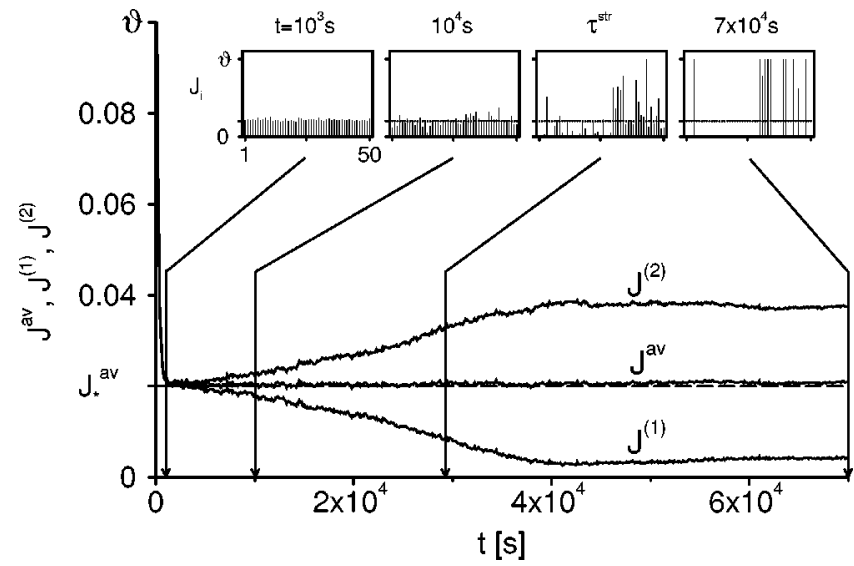

FIG. 9. Temporal evolution of average weights $J^{\text {av }}, J^{(1)}$, and $J^{(2)}$ as a function of the learning time $t$ in units of $10^{4} \mathrm{~s}$. The quantity $J^{\text {av }}$ is the average weight of all synapses, $J^{(1)}$ and $J^{(2)}$ are average weights in groups $\mathcal{M}_{1}$ and $\mathcal{M}_{2}$, respectively. Synapses $i$ in group $\mathcal{M}_{1}$, where $1 \leqslant i \leqslant 25$, receive incoherent input, whereas synapses $i$ in group $\mathcal{M}_{2}$, where $26 \leqslant i \leqslant 50$, are driven by a coherently modulated input intensity. Parameters are as given in Appendix B. Simulations started at time $t=0$ with a homogeneous weight distribution $J_{i}=\vartheta=0.1$ for all $i$. The normalization of the average weights takes place within a time of order $O(100 \mathrm{~s})$; see also the uppermost full line in Fig. 8. On the time scale of $\tau^{\mathrm{str}}=2.93$ $\times 10^{4} \mathrm{~s}$ a structure in the distribution of weights emerges in that $J^{(2)}$ grows at the expense of $J^{(1)}$. The average weight $J^{\text {av }}$ remains almost unaffected near $J_{*}^{\text {av }}=2 \times 10^{-2}$ (dashed line). The slight enlargement of $J^{\text {av }}$ between $t=10^{4} \mathrm{~s}$ and $t=7 \times 10^{4} \mathrm{~s}$ can be explained by using Eq. (C1) and taking also the $k_{3}$ term into account. The insets (signatures as in Figs. 7 and 8) show the weight distributions at times $t=10^{3}, 10^{4}, 2.93 \times 10^{4}$, and $7 \times 10^{4} \mathrm{~s}$ (arrows).

Since we only need an estimate of $\tau^{\text {str }}$ we drop the logarithm, which is of order 1 . Finally, approximating $N /\left(M_{1} M_{2}\right)$ by $1 / N$ we arrive at the estimate

$$
\tau^{\mathrm{str}}=(N Q)^{-1} .
$$

We could adopt a refined analysis similar to the one we have used for $J^{\text {av }}$ to discuss the effects of the upper and lower bounds for individual weights. We will not do so, however, since the result (29) suffices for our purpose: the comparison of time constants.

A comparison of $\tau^{\text {av }}$ in Eq. (22) with $\tau^{\text {str }}$ in Eq. (29) shows that we have a separation of the fast time scale of normalization from the slow time scale of structure formation, if Eq. (23) holds.

A numerical example confirming the above theoretical considerations is presented in Fig. 9. Simulation parameters are as given in Appendix B.

\section{Stabilization of learning}

Up to this point we have neglected the influence of the $k_{3}$ term in Eq. (15), which may lead to a stabilization of weight distributions, in particular when synapses are few and strong $[22,54]$; cf. Sec. IV D. This is the case, for example, in the scenario of Fig. 10, which is the final result of the simulations described in Fig. 9. The shown weight distribution is stable so that learning has terminated apart from minor rapid fluctuations due to noise.

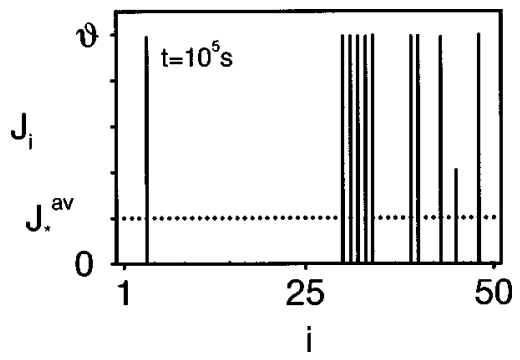

FIG. 10. The asymptotic distribution of weights $\left\{J_{i}\right\}$ at time $t$ $=10^{5} \mathrm{~s}$; signatures are as in Fig. 7. This distribution is the final result of the numerical simulation shown in Fig. 9 and remains stable thereafter apart from minor rapid fluctuations. All but one of the synapses are saturated either at the lower or at the upper bound.

In stable weight distributions, it is now shown that all synapses but one are saturated either at the lower or the upper bound. In the scenario of Fig. 10, the $k_{3}$ term keeps a single weight $J_{m_{1}}$ in group $\mathcal{M}_{1}$ at the upper bound $\vartheta$, even though there is a nonsaturated one $J_{m_{2}}$ in $\mathcal{M}_{2}$. Group $\mathcal{M}_{2}$ (in contrast to $\mathcal{M}_{1}$ ) comprises most of the total weight and is driven by positively correlated input. Why does $J_{m_{1}}$ not decrease in favor of $J_{m_{2}}$ ? The answer comes from Eq. (15). Weight $J_{m_{1}}$ receives a stronger reinforcement than $J_{m_{2}}$, if $\dot{J}_{m_{1}}>\dot{J}_{m_{2}}$ holds. Using Eq. (15) we find $J_{m_{1}}$ $>Q / k_{3} \Sigma_{j \in \mathcal{M}_{2}} J_{j}+J_{m_{2}} . \quad$ Approximating $\quad \Sigma_{j \in \mathcal{M}_{2}} J_{j}=N J_{*}^{\mathrm{av}}$ $=-k_{1} / k_{2}$ we obtain $J_{m_{1}}>-Q k_{1} /\left(k_{2} k_{3}\right)+J_{m_{2}}$. This condition is fulfilled because $J_{m_{1}} \approx 0.1, J_{m_{2}} \approx 0.04$ (cf. Fig. 10), and $-Q k_{1} /\left(k_{2} k_{3}\right) \approx 0.01$ (cf. Table I); here $k_{1}>0, k_{2}<0$, and $k_{3}>0$.

\section{NOISE}

In this section we discuss the influence of noise on the evolution of each weight. Noise may be due to jitter of input and output spikes and the fact that we deal with spikes per se (Sec. II B). This gives rise to a random walk of each weight around the mean trajectory described by Eq. (15). The variance $\operatorname{var} J_{i}(t)$ of this random walk increases linearly with time as it does in free diffusion. From the speed of the variance increase we derive a time scale $\tau^{\text {noise }}$. A comparison with the time constant $\tau^{\text {str }}$ of structure formation leads to further constraints on our learning parameters and shows that, in principle, any correlation in the input, however weak, can be learned, if there is enough time available for learning.

The calculation of $\operatorname{var} J_{i}$ is based on four approximations. First, we neglect upper and lower bounds of the learning dynamics as we have done for the calculation of the time constants of normalization (Sec. V B) and structure formation (Sec. V C). Second, we neglect spike-spike correlations between input and output and work directly with ensembleaveraged rates. As we have seen, spike-spike correlations show up in the term $k_{3}$ in Eq. (15) and have little influence on learning, given many, weak synapses and an appropriate scenario for our learning parameters; cf. Sec. IV C. Third, we assume constant input rates $\lambda_{i}^{\text {in }}(t)=\nu^{\text {in }}$ for all $i$. A temporal structure in the input rates is expected to play a minor role here. Fourth, as a consequence of constant input rates we assume a constant output rate $\lambda^{\text {out }}(t)=\nu^{\text {out }}$. 
Despite such a simplified approach, we can study some interesting effects caused by neuronal spiking. Within the limits of our approximations, input and output spikes are generated by independent Poisson processes with constant intensities. The variance $\operatorname{var} J_{i}(t)$ increases basically because of shot noise at the synapses. We now turn to the details.

\section{A. Calculation of the variance}

We start with some weight $J_{i}\left(t_{0}\right)$ at time $t_{0}$ and calculate the variance $\operatorname{var} J_{i}(t):=\left\langle J_{i}^{2}\right\rangle(t)-\left\langle J_{i}\right\rangle^{2}(t)$ as a function of $t$ for $t>t_{0}$. Angular brackets \langle\rangle again denote an ensemble average; cf. Sec. II B. A detailed analysis is outlined in Appendix $\mathrm{E}$. The result is

$$
\operatorname{var} J_{i}(t)=\left(t-t_{0}\right) D \text { for } t-t_{0} \gg \mathcal{W},
$$

where $\mathcal{W}$ is the width of the learning window $W$ (cf. Sec. II C) and

$$
\begin{aligned}
D= & \nu^{\text {in }}\left(w^{\text {in }}\right)^{2}+\nu^{\text {out }}\left(w^{\text {out }}\right)^{2}+\nu^{\text {in }} \nu^{\text {out }} \int d s W(s)^{2} \\
& +\nu^{\text {in }} \nu^{\text {out }} \widetilde{W}(0)\left[2\left(w^{\text {in }}+w^{\text {out }}\right)+\widetilde{W}(0)\left(\nu^{\text {in }}+\nu^{\text {out }}\right)\right] .
\end{aligned}
$$

Thus because of Poisson spike arrival and stochastic output firing with disjoint intervals being independent, each weight $J_{i}$ undergoes a diffusion process with diffusion constant $D$.

To discuss the dependence of $D$ upon the learning parameters, we restrict our attention to the case $\nu^{\text {in }}=\nu^{\text {out }}$ in Eq. (31). Since mean input and output rates in biological neurons typically are not too different, this makes sense. Moreover, we do not expect that the ratio $\nu^{\text {in }} / \nu^{\text {out }}$ is a critical parameter. We recall from Sec. V B that $\nu^{\text {out }}=-k_{1} / k_{2} \nu^{\text {in }}$ once the weights are already normalized and if $\nu_{0}=Q^{\text {av }}=0$. With $\nu^{\text {in }}=\nu^{\text {out }}$ this is equivalent to $k_{1}=-k_{2}$. Using the definition of $k_{1}$ and $k_{2}$ in Eq. (14) we find $w^{\text {in }}+w^{\text {out }}=-\widetilde{W}(0) \nu^{\text {in }}$. If we insert this into Eq. (31), the final term vanishes. In what remains of Eq. (31) we identify the contributions due to input spikes, $\nu^{\text {in }}\left(w^{\text {in }}\right)^{2}$, and output spikes, $\nu^{\text {out }}\left(w^{\text {out }}\right)^{2}$. Weight changes because of correlations between input and output spikes enter Eq. (31) via $\nu^{\text {in }} \nu^{\text {out }} \int d s W(s)^{2}$.

Equation (30) describes the time course of the variance of a single weight. Estimating $\operatorname{var} J_{i}$ is numerically expensive because we have to simulate many independent learning trials. It is much cheaper to compute the variance of the distribution $\left\{J_{i}\right\}$ of weights in a single learning trial. For the sake of a comparison of theory and numerics in Fig. 11, we plot

$$
\operatorname{var}\left\{J_{i}\right\}(t):=\frac{1}{N-1} \sum_{i=1}^{N}\left[J_{i}(t)-J^{\mathrm{av}}(t)\right]^{2},
$$

which obeys a diffusion process with

$$
\operatorname{var}\left\{J_{i}\right\}(t)=\left(t-t_{0}\right) D^{\prime},
$$

in a way similar to Eq. (30). The diffusion constant $D^{\prime}$ is, however, different from $D$ because weights of single neurons do not develop independently of each other. Each output spike triggers the change of all $N$ weights by an amount $w^{\text {out }}$. Therefore, output spikes do not contribute to a change of

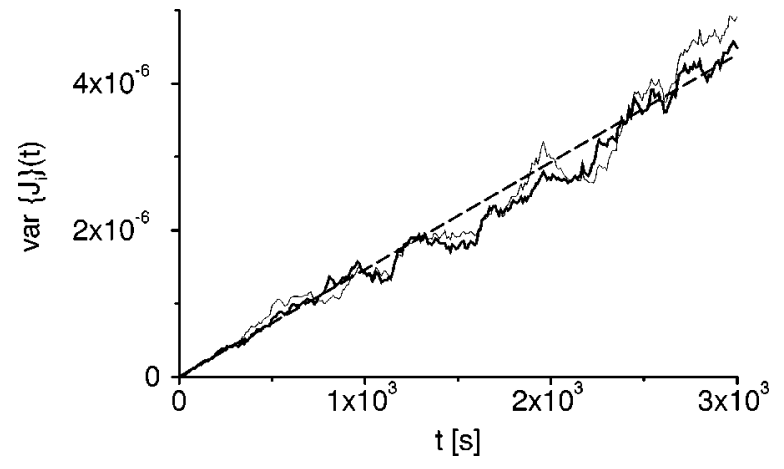

FIG. 11. Influence of noise. We compare numerical results for the evolution of the variance $\operatorname{var}\left\{J_{i}\right\}(t)$ defined in Eq. (32) (full lines) with our theoretical prediction (dashed line) based on Eq. (33) with $D^{\prime}=1.47 \times 10^{-9} \mathrm{~s}^{-1}$. Learning starts at time $t=0$ with a homogeneous distribution, $J_{i}=J_{*}^{\mathrm{av}}=0.02$ for all $i$. The thin line corresponds to the simulation of Fig. 8 with initial condition $J^{\text {av }}$ $=0.02$, viz., two groups of 25 synapses each. The thick line has been obtained with incoherent input for all 50 synapses, $\lambda_{i}^{\text {in }}(t)$ $=\nu^{\text {in }}$ for all $i$ (all other parameters in Appendix B being equal). Because the number of synapses is finite $(N=50)$, deviations from the dashed straight line are due to fluctuations. The overall agreement between theory and simulation is good.

$\operatorname{var}\left\{J_{i}\right\}(t)$ as long as upper and lower bounds have not been reached. Furthermore, all synapses "see" the same spike train of the postsynaptic neuron they belong to. In contrast to that, input spikes at different synapses are independent. Again we assume that input and output spikes are independent; cf. the second paragraph at the beginning of Sec. VI. Combining the above arguments, we obtain the diffusion constant $D^{\prime}$ by simply setting $w^{\text {out }}=0$ and disregarding the term $\left[\nu^{\text {in }} \widetilde{W}(0)\right]^{2} \nu^{\text {out }}$ in Eq. (31), which leads to $D^{\prime}=\nu^{\text {in }}\left(w^{\text {in }}\right)^{2}+\nu^{\text {in }} \nu^{\text {out }}\left[\int d s W(s)^{2}+2 w^{\text {in }} \widetilde{W}(0)+\widetilde{W}(0)^{2}\right]$. The boundaries of validity of Eq. (33) are illustrated in Fig. 12 .

\section{B. Time scale of diffusion}

The effects of shot noise in input and output show up on a time scale $\tau^{\text {noise }}$ which may be defined as the time interval necessary for an increase of the variance (30) from $\operatorname{var} J_{i}\left(t_{0}\right)=0$ to $\operatorname{var} J_{i}\left(t_{0}+\tau^{\text {noise }}\right)=\left(J_{*}^{\text {av }}\right)^{2}$. We chose $J_{*}^{\text {av }}$ as a reference value because it represents the available range for each weight. From Eq. (30) we obtain $\tau^{\text {noise }}=\left(J_{*}^{\text {av }}\right)^{2} / D$. We use $J_{*}^{\text {av }}=-k_{1} /\left(N k_{2}\right)$ from Eq. (21) and $Q^{\text {av }}=0$. This yields

$$
\tau^{\text {noise }}=\frac{1}{N^{2} D}\left(\frac{k_{1}}{k_{2}}\right)^{2}
$$

\section{Comparison of time scales}

We now compare $\tau^{\text {noise }}$ in Eq. (34) with the time constant $\tau^{\mathrm{str}}=1 /(N Q)$ of structure formation as it appears in Eq. (29). The ratio

$$
\frac{\tau^{\mathrm{noise}}}{\tau^{\mathrm{str}}}=\frac{Q}{N D}\left(\frac{k_{1}}{k_{2}}\right)^{2}
$$

should exceed 1 so as to enable structure formation (in the sense of Sec. V C). Otherwise weight diffusion due to noise spreads the weights between the lower bound 0 and the upper bound $\vartheta$ and, consequently, destroys any structure. 


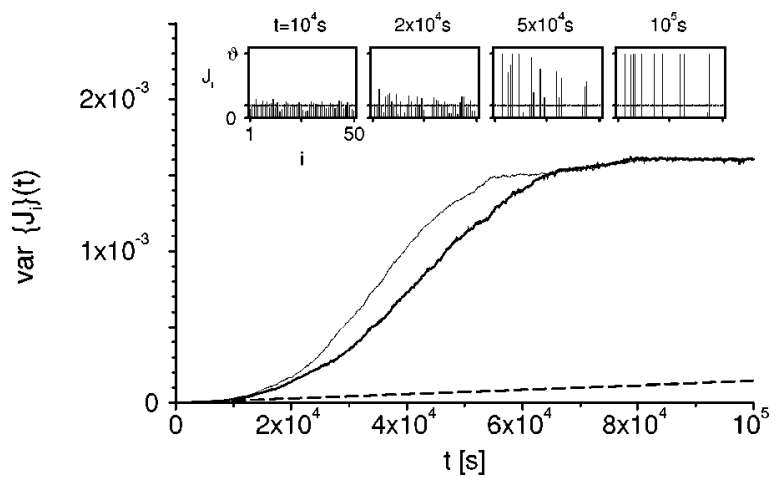

FIG. 12. The variance var $\left\{J_{i}\right\}(t)$ as in Fig. 11 but on a longer time scale. Thick line: all 50 synapses receive incoherent input. Thin line: two groups of synapses that are treated differently, as in Figs. 8, 9, and 10. The four insets (signatures as in Fig. 7) correspond to the thick line scenario and show the evolution of the distribution of synaptic weights. As in Fig. 11, full lines are numerical results and the dashed line is our theoretical prediction. Both differ significantly for times $t>10^{4} \mathrm{~s}$. The reason is that Eq. (33) does not include the influence of correlations between input and output. Spike-spike correlations due to the $k_{3}$ term increase weights with a velocity proportional to their weight; cf. Eq. (15). Large weights, which are already present at times $t \geq 2 \times 10^{4} \mathrm{~s}$ (see inset), therefore grow at the expense of the smaller ones. This gives rise to an enlarged variance (thick full line). In the thin-line scenario, we also have the $Q_{i j}$ term in Eq. (15), which contributes to an additional increase of $\operatorname{var}\left\{J_{i}\right\}$. Finally, at $t \approx 10^{5} \mathrm{~s}, \operatorname{var}\left\{J_{i}\right\}$ saturates because most of the weights are either at the lower or at the upper bound.

We note that $D$ in Eq. (35) is quadratic in $w^{\text {in }}, w^{\text {out }}$, and $W$, whereas $k_{1}, k_{2}$, and $Q$ are linear; cf. Eqs. (12), (14), (17), and (31). As a consequence, scaling $w^{\text {in }}, w^{\text {out }}$, and $W(s)$ (or the learning parameter $\eta$ ) in Eq. (1) by a common factor $\gamma$ changes the ratio of time constants in Eq. (35) by $1 / \gamma$ without affecting the (normalized) mean output rate and the fixed points $J_{*}^{\mathrm{av}}$ and $J_{*}^{\mathrm{str}}=-J_{*}^{\mathrm{av}} N / M_{1}$; cf. Eq. (21). Hence it is always possible to achieve $\tau^{\text {noise }} / \tau^{\text {str }}>1$ by tuning $\gamma$. This means that any covariance matrix (11) that gives rise to $Q>0$, however small, can be learned. More precisely, it can be learned if there is enough time for learning.

A reduction of $\gamma$ also increases the time constant $\tau^{\text {str }}$ $=1 /(N Q)$ of structure formation; cf. Eq. (29). If the learning time is limited, which may be the case in biological systems, only input with $Q$ larger than some minimal value can be learned. Considering the learning parameters as fixed, we see that increasing the number of synapses, on the one hand, helps reduce the time $\tau^{\mathrm{str}}$ necessary for learning but, on the other hand, decreases the ratio $\tau^{\text {noise }} / \tau^{\text {str }}$ in Eq. (35), possibly below 1.

With parameters as given in Appendix B, the ratio (35) is 5.5. Therefore, the desired structure in Fig. 9 can emerge before noise spreads the weights at random.

\section{DISCUSSION}

Changes of synaptic efficacies are triggered by the relative timing of presynaptic and postsynaptic spikes [25,26]. The learning rule (1) discussed in this paper is a first step towards a description and analysis of the effects of synaptic changes with single-spike resolution. Our learning rule can be motivated by elementary dynamic processes at the level of the synapse $[54,57]$ and can also be implemented in hardware; cf. [40]. A phenomenological model of the experimental effects which is close to the model studied in the present paper has been introduced [42]. A compartmental model of the biophysics and ion dynamics underlying spike-based learning along the lines of [58] has not been attempted yet. As an alternative to changing synaptic weights, spike-based learning rules which act directly on the delays may also be considered [59-61].

The learning rule (1) discussed in the present paper is rather simple and contains only terms that are linear and quadratic in the presynaptic and postsynaptic spikes ("Hebbian" learning). This simple mathematical structure, which is based on experiment $[25,26,30]$, has allowed us to derive analytical results and identify some key quantities.

First of all, if the input signal contains no correlations with the output at the spike level, and if we use a linear Poissonian neuron model, the spike-based learning rule reduces to Eq. (15), which is closely reminiscent of Linsker's linear learning equation for rate coding [3]. The only difference is an additional term $k_{3}$, which is not accounted for by pure rate models. It is caused by precise temporal correlations between an output spike and an input spike that has triggered the pulse. This additional term reinforces synapses that are already strong and hence helps to stabilize existing synapse configurations.

In the limit of rate coding, the form of the learning window $W$ is not important but only the integral $\int d s W(s)$ counts: $\int d s W(s)>0$ would be called "Hebbian," $\int d s W(s)<0$ is sometimes called "anti-Hebbian" learning. In general, however, input rates may be modulated on a fast time scale or contain correlations at the spike level. In this case, the shape of the learning window does matter. A learning window with a maximum at $s^{*}<0$ (thus maximal increase of the synaptic strength for a presynaptic spike preceding a postsynaptic spike; cf. Fig. 6) picks up the correlations in the input. In this case a structured distribution of synaptic weights may evolve [22].

The mathematical approach developed in this paper leads to a clear distinction between different time scales. First, the fastest time scale is set by the time course of the postsynaptic potential $\epsilon$ and the learning window $W$. Correlations in the input may occur on the same fast time scale, but can also be slower or faster, there is no restriction. Second, learning occurs on a much slower time scale and in two phases: (i) an intrinsic normalization of total synaptic weight and the output firing rate followed by (ii) structure formation. Third, if the learning rate is small enough, then diffusion of the weights due to noise is slow as compared to structure formation. In this limit, the learning process is described by the differential equation (4) for the expected weights.

Normalization is possible, if at least $w^{\text {in }}>0$ and $w^{\text {out }}<0$ for $\int d s W(s)>0$ in Eq. (1) ("Hebbian" learning). In this case, the average weight may decay exponentially to a fixed point, though there is no decay term for individual weights. In other words, normalization is an intrinsic property since we do not invoke multiplicative or subtractive rescaling of weights after each learning step $[2,7,55]$.

The fluctuations due to noise have been treated rather crudely in the present paper. In principle, it should be pos- 
sible to include the effects of noise directly at the level of the differential equation, as is standard in statistics [62]. Such an approach would then lead to a Fokker-Planck equation for the evolution of weights as discussed in [63]. All this is in principle straightforward but in practice very cumbersome.

Finally, we emphasize that we have used a crudely oversimplified neuron model, viz., a linear stochastic unit. In particular, there is no spike emission threshold nor reset or spike afterpotential. Poisson firing is not as unrealistic as it may at first seem, though. Large networks of integrate-and-fire neurons with stochastic connectivity exhibit Poisson-like firing [64]. Experimental spike interval distributions are also consistent with Poisson firing [65]. In the present paper, the simple Poisson model has been chosen so as to grasp the mathematics and get an explicit expression for the correlation between input and output spikes. The formulation of the learning rule and the derivation of the learning equation (4) is general and holds for any neuron model. The calculation of the correlations which enter in the definition of the parameter $Q_{i j}$ in Eq. (15) is, however, much more difficult, if a nonlinear neuron model is used.

Spike-based Hebbian learning has important implications for the question of neural coding since it allows us to pick up and stabilize fast temporal correlations [38,22,41]. A better understanding of spike-triggered learning may thus also contribute to a resolution of the problem of neural coding [17,19,65-67].

\section{ACKNOWLEDGMENTS}

The authors thank Christian Leibold for helpful comments and a careful reading of the manuscript. R.K. has been supported by the Deutsche Forschungsgemeinschaft (DFG) under Grant Nos. He 1729/8-1 and Kl 608/10-1 (FG Hörobjekte). The authors also thank the DFG for travel support (Grant No. He 1729/8-1).

\section{APPENDIX A: PROOF OF EQ. (9)}

In proving Eq. (9) there is no harm in setting $\nu_{0}=0$. We then have to compute the average

$\left\langle S_{i}^{\text {in }}(t+s) S^{\text {out }}(t)\right\rangle=\left\langle S_{i}^{\text {in }}(t+s)\left[f_{i}(t)+\sum_{j(\neq i)} f_{j}(t)\right]\right\rangle$,

where $f_{i}(t)=J_{i}(t) \Sigma_{f} \epsilon\left(t-t_{i}^{f}\right)$ with the upper index $f$ ranging over the firing times $t_{i}^{f}<t$ of neuron $i$, which has an axonal connection to synapse $i$; here $1 \leqslant i \leqslant N$. Since $\epsilon$ is causal, i.e., $\epsilon(s) \equiv 0$ for $s<0$, we can drop the restriction $t_{i}^{f}<t$. The synapses being independent of each other, the sum over $j$ $(\neq i)$ is independent of $S_{i}^{\text {in }}$ and thus we obtain

$$
\begin{aligned}
\left\langle S_{i}^{\mathrm{in}}(t+s)\left[\sum_{j(\neq i)} f_{j}(t)\right]\right\rangle \\
=\left\langle S_{i}^{\mathrm{in}}\right\rangle(t+s)\left[\sum_{j(\neq i)}\left\langle f_{j}\right\rangle(t)\right] \\
=\lambda_{i}^{\mathrm{in}}(t+s)\left[\sum_{j(\neq i)} J_{j}(t) \int_{0}^{\infty} d t^{\prime} \epsilon\left(t^{\prime}\right) \lambda_{j}^{\mathrm{in}}\left(t-t^{\prime}\right)\right] .
\end{aligned}
$$

The term $\left\langle S_{i}^{\text {in }}(t+s) f_{i}(t)\right\rangle$ in Eq. (A1) has to be handled with more care as it describes the influence of synapse $i$ on the firing behavior of the postsynaptic neuron,

$$
\begin{aligned}
\left\langle S_{i}^{\mathrm{in}}(t+s)\right. & \left.f_{i}(t)\right\rangle \\
& =\left\langle\left[\sum_{f^{\prime}} \delta\left(t+s-t_{i}^{f^{\prime}}\right)\right]\left[J_{i}(t) \sum_{f} \epsilon\left(t-t_{i}^{f}\right)\right]\right\rangle .
\end{aligned}
$$

The first term on the right in Eq. (A3) samples spike events at time $t+s$. To be mathematically precise, we sample all spikes in a small interval of size $\Delta t$ around $t+s$, average, and divide by $\Delta t$. We replace the first sum in Eq. (A3) by the (approximate) identity $(\Delta t)^{-1} \mathbb{1}_{\text {spike in }[t+s, t+s+\Delta t)\}}$, where $1_{\{\}}$is the indicator function of the set \{\} ; i.e., it equals 1 when its argument is in the set \{\} and 0 elsewhere. Because the postsynaptic potential $\epsilon$ is a continuous function, we approximate the second sum by $\Sigma_{k} 1_{\left.\text {\{spike in }\left[t_{k}, t_{k}+\Delta t\right)\right\}} \boldsymbol{\epsilon}\left(t-t_{k}\right)$, where $\left\{\left[t_{k}, t_{k}+\Delta t\right), k \in \mathbb{Z}\right\}$ is a decomposition of the real axis. Since it is understood that $\Delta t \rightarrow 0$, all events with two or more spikes in an interval $\left[t_{k}, t_{k}+\Delta t\right)$ have a probability $o(\Delta t)$ and, hence, can be neglected. It is exactly this property that is typical to a Poisson process - and to any biological neuron.

What we are going to compute is the correlation between $S_{i}^{\text {in }}$, the input at synapse $i$, and the output $S^{\text {out }}$, which is governed by all synapses, including synapse $i$. Here the simplicity of the linear Poissonian neuron model pays off as $S^{\text {out }}$ is linear in the sum of the synaptic inputs and, hence, in each of them. Furthermore, whatever the model, the synaptic efficacies $J_{i}(t)$ are changing adiabatically with respect to the neuronal dynamics so that they can be taken to be constant and, thus, out of the average. In the limit $\Delta t \rightarrow 0$ we can therefore rewrite the right-hand side of Eq. (A3) so as to find

$$
\begin{aligned}
J_{i}(t) & (\Delta t)^{-1} \sum_{k} \epsilon\left(t-t_{k}\right) \\
& \left.\times\left\langle\mathbb{1}_{\{\text {spike in }[t+s, t+s+\Delta t)\}} \mathbb{1}_{\left\{\text {spike in }\left[t_{k}, t_{k}+\Delta t\right)\right.}\right\}\right\rangle .
\end{aligned}
$$

Without restriction of generality we can choose our partition so that $t_{k}=s+t$ for some $k$, say $k=l$. Singling out $k$ $=l$, the rest $(k \neq l)$ can be averaged directly, since events in disjoint intervals are independent. Because $\left\langle 1_{\{\}}\right\rangle$ $=\operatorname{prob}\{$ spike in $[t+s, t+s+\Delta t)\}=\lambda_{i}^{\text {in }}(t+s) \Delta t$, the result is $J_{i}(t) \lambda_{i}^{\text {in }}(t+s) \Lambda_{i}^{\mathrm{in}}(t)$, where we have used Eq. (8). As for the term $k=l$, we plainly have $1_{\{\}}^{2}=l_{\{\}}$, as an indicator function assumes only two distinct values, 0 and 1 . We obtain $J_{i}(t) \lambda_{i}^{\text {in }}(t+s) \epsilon(-s)$.

Collecting terms and incorporating $\nu_{0} \neq 0$, we find Eq. (9). 


\section{APPENDIX B: PARAMETERS FOR NUMERICAL SIMULATIONS}

We discuss the parameter regime of the simulations as shown in Secs. V and VI. Numerical values and important derived quantities are summarized in Table I.

\section{Learning window}

We use the learning window

$$
W(s)=\eta\left\{\begin{array}{l}
\exp \left(\frac{s}{\tau^{\text {syn }}}\right)\left[A_{+}\left(1-\frac{s}{\tilde{\tau}_{+}}\right)+A_{-}\left(1-\frac{s}{\tilde{\tau}_{-}}\right)\right] \quad \text { for } s \leqslant 0, \\
A_{+} \exp \left(-\frac{s}{\tau_{+}}\right)+A_{-} \exp \left(-\frac{s}{\tau_{-}}\right) \quad \text { for } s>0 .
\end{array}\right.
$$

Here $s$ is the delay between presynaptic spike arrival and postsynaptic firing, $\eta$ is a "small" learning parameter, $\tau^{\mathrm{syn}}, \tau_{+}, \tau_{-}, \tilde{\tau}_{+}:=\tau^{\mathrm{syn}} \tau_{+} /\left(\tau^{\mathrm{syn}}+\tau_{+}\right)$, and $\tilde{\tau}_{-}:=\tau^{\mathrm{syn}} \tau_{-} /\left(\tau^{\mathrm{syn}}\right.$ $\left.+\tau_{-}\right)$are time constants. The dimensionless constants $A_{+}$ and $A_{-}$determine the strength of synaptic potentiation and depression, respectively. Numerical values are $\eta$ $=10^{-5}, \tau^{\text {syn }}=5 \mathrm{~ms}, \tau_{+}=1 \mathrm{~ms}, \tau_{-}=20 \mathrm{~ms}$, and $A_{+}$ $=1, A_{-}=-1$; cf. Table I. The learning window (cf. Fig. 6) is in accordance with experimental results [25,26,28,29,33]. A detailed explanation of our choice of the learning window on a microscopical basis of Hebbian learning can be found elsewhere [54,57].

For the analysis of the learning process we need the integrals $\widetilde{W}(0):=\int d s W(s)$ and $\int d s W(s)^{2}$. The numerical result is listed in Table I. Using $c_{+}:=\tau^{\mathrm{syn}} / \tau_{+}$and $c_{-}:=\tau^{\mathrm{syn}} / \tau_{-}$we obtain

$$
\begin{aligned}
\int d s W(s)= & \eta \tau^{\mathrm{syn}}\left[A_{-}\left(2+c_{-}+c_{-}^{-1}\right)\right. \\
& \left.+A_{+}\left(2+c_{+}+c_{+}^{-1}\right)\right]
\end{aligned}
$$

and

$$
\begin{aligned}
\int d s W(s)^{2}= & \frac{\eta^{2}}{4}\left\{A_{-}^{2} \tau_{-}\left[c_{-}^{3}+4 c_{-}^{2}+5 c_{-}+2\right]\right. \\
& +A_{+}^{2} \tau_{+}\left[c_{+}^{3}+4 c_{+}^{2}+5 c_{+}+2\right] \\
& +2 A_{+} A_{-} \tau^{\mathrm{syn}}\left[c_{+} c_{-}+2\left(c_{+}+c_{-}\right)\right. \\
& \left.\left.+5+4 /\left(c_{+}+c_{-}\right)\right]\right\}
\end{aligned}
$$

\section{Postsynaptic potential}

We use the excitatory postsynaptic potential (EPSP)

$$
\epsilon(s)=s / \tau_{0}^{2} \exp \left(-s / \tau_{0}\right) \mathcal{H}(s),
$$

where $\mathcal{H}()$ denotes the Heaviside step function, and $\int d s \epsilon(s)=1$. For the membrane time constant we use $\tau_{0}$ $=10 \mathrm{~ms}$, which is reasonable for cortical neurons $[68,69]$. The EPSP has been plotted in Fig. 4. Using Eqs. (B1) and (B4) we obtain

$$
\begin{aligned}
\int d s W(s) \epsilon(-s)= & \eta\left(\tau^{\mathrm{syn}}\right)^{2} /\left(\tau^{\mathrm{syn}}+\tau_{0}\right)^{3} \\
& \times\left[A_{-}\left(2 \tau^{\mathrm{syn}} \tau_{0} / \tau_{-}+\tau^{\mathrm{syn}}+3 \tau_{0}\right)\right. \\
& \left.+A_{+}\left(2 \tau^{\mathrm{syn}} \tau_{0} / \tau_{+}+\tau^{\mathrm{syn}}+3 \tau_{0}\right)\right]
\end{aligned}
$$

\section{Synaptic input}

The total number of synapses is $N=50$. For $1 \leqslant i \leqslant M_{1}$ $=25$ synapses in group $\mathcal{M}_{1}$ we use a constant input intensity $\lambda_{i}^{\text {in }}(t)=\nu^{\text {in }}$. The remaining $M_{2}=25$ synapses receive a periodic intensity, $\lambda_{i}^{\text {in }}(t)=\nu^{\text {in }}+\delta \nu^{\text {in }} \cos (\omega t)$ for $i \in \mathcal{M}_{2}$; cf. also Sec. V A 3. Numerical parameters are $\nu^{\text {in }}=10 \mathrm{~Hz}, \delta \nu^{\text {in }}$ $=10 \mathrm{~Hz}$, and $\omega /(2 \pi)=40 \mathrm{~Hz}$. For the comparison of theory and simulation we need the value of $Q$ in Eq. (18). We numerically took the Fourier transforms of $\epsilon$ and $W$ at the frequency $\omega$. The time constant $\tau^{\text {str }}$ is calculated via Eq. (29); cf. Table I.

\section{Parameters $w^{\text {in }}, w^{\text {out }}, \nu_{0}$, and $\vartheta$}

We use the learning parameters $w^{\text {in }}=\eta$ and $w^{\text {out }}$ $=-1.0475 \eta$, where $\eta=10^{-5}$. The spontaneous output rate is $\nu_{0}=0$ and the upper bound for synaptic weights is $\vartheta$ $=0.1$. These values have been chosen in order to fulfill the following five conditions for learning. First, the absolute values of $w^{\text {in }}$ and $w^{\text {out }}$ are of the same order as the amplitude of the learning window $W$; cf. Fig. 6. Furthermore, these absolute values are small as compared to the normalized average weight (see below). Second, the constraints on $k_{1}$ and $k_{2}$ for a stable and realizable fixed point are satisfied; cf. Sec. V B and Eq. (14). Third, the correlations in the input are weak so that $0<Q \ll-k_{2}$; cf. Eq. (23). This implies that the time scale $\tau^{\text {av }}$ of normalization in Eq. (22) is orders of magnitude smaller than the time scale $\tau^{\text {str }}$ of structure formation in Eq. (29); cf. also Table I. Fourth, the $k_{3}$ term in Eq. (14) can be neglected in the sense of Sec. IV C. Proving this, we note that the fixed point for the average weight is $J_{*}^{\text {av }}=2 \times 10^{-2}$ [cf. Eq. (21)] and $k_{3}=7.04 \times 10^{-5} \mathrm{~s}^{-1}$. We now focus on Eq. (16). Since $Q_{i j}\left(\ll\left|k_{2}\right|\right.$ for all $\left.i, j\right)$ can be neglected and $J_{i} \leqslant \vartheta$ for all $i$, we find from Eq. (16) the even more restrictive condition $N\left|k_{2}\right| J_{*}^{\text {av }} / \vartheta \gg\left|k_{3}\right|$ which is fulfilled in our pa- 
rameter regime. Fifth, input and output rates are identical for normalized weights, $\nu^{\text {in }}=\nu^{\text {out }}$ for $\nu_{0}=0$; see Sec. V B.

\section{APPENDIX C: NORMALIZATION AND CORRELATIONS BETWEEN WEIGHTS AND INPUT RATES}

The assumption of independence of the weights $\left\{J_{i}\right\}$ and the rates $\left\{\lambda_{i}^{\text {in }}\right\}$ used in Sec. V B for the derivation of a normalization property of Eq. (15) is not valid in general. During learning we expect weights to change according to their input. For the configuration of the input as introduced in Sec. V A this depends on whether synapses belong to groups $\mathcal{M}_{1}$ or $\mathcal{M}_{2}$. To show that even under the condition of interdependence of $\left\{J_{i}\right\}$ and $\left\{\lambda_{i}^{\text {in }}\right\}$ there is a normalization property of Eq. (15) similar to that derived in Sec. V B, we investigate the most extreme case in which the total mass of synaptic weight is, e.g., in $\mathcal{M}_{2}$. Taking $J_{i}=0$ for $i \in \mathcal{M}_{1}$ into account, we replace $N^{-1} \sum_{i j} J_{j} Q_{i j}$ in Eq. (20) by $M_{2}^{-1} N^{2} J^{\text {av }} Q^{\text {av }}$. The fixed point $J_{*}^{\text {av }}$ is similar to that in Eq. (21) except for a multiplicative prefactor $N / M_{2}$ of order 1 preceding $Q^{\text {av }}$ in Eq. (21),

$$
J_{*}^{\mathrm{av}}=-k_{1} /\left[N\left(k_{2}+Q^{\mathrm{av}} N / M_{2}\right)\right] .
$$

Since $N / M_{2}>1, k_{1}>0$, and $k_{2}+Q^{\mathrm{av}} N / M_{2}<0, J_{*}^{\mathrm{av}}$ in Eq. (C1) is larger than $J_{*}^{\text {av }}$ in Eq. (21), where we assumed independence of $\left\{J_{i}\right\}$ and $\left\{\lambda_{i}^{\text {in }}\right\}$. Correlations between $\left\{J_{i}\right\}$ and $\left\{\lambda_{i}^{\text {in }}\right\}$ can be neglected, however, if we assume $0<Q \approx Q^{\text {av }}$ $\ll-k_{2}$; cf. Eq. (23). In this case, $J_{*}^{\text {av }}$ in Eqs. (21) and (C1) are almost identical and independent of $Q^{\text {av }}$.

\section{APPENDIX D: NORMALIZATION AND WEIGHT CONSTRAINTS}

Let us consider the influence of weight constraints (Sec. V B) on the position of the fixed point $J_{*}^{\text {av }}$ in Eq. (21) and the time constant $\tau^{\text {av }}$ of normalization in Eq. (22). We call $N_{\downarrow}$ and $N_{\uparrow}$ the number of weights at the lower bound 0 and the upper bound $\vartheta>0$, respectively. By construction we have $N_{\downarrow}+N_{\uparrow} \leqslant N$, where $N$ is the number of synapses.

For example, if the average weight $J^{\text {av }}$ approaches $J_{*}^{\text {av }}$ from below, then only $N-N_{\uparrow}$ weights can contribute to an increase of $J^{\text {av }}$. For the remaining $N_{\uparrow}$ saturated synapses we have $\dot{J}_{i}=0$. Deriving from Eq. (15) an equation equivalent to Eq. $\quad(20)$, we obtain $\dot{J}^{\text {av }}=\left(1-N_{\uparrow} / N\right)\left(k_{1}+N k_{2} J^{\text {av }}\right.$ $\left.+J^{\text {av }} Q^{\text {av }} / N\right)$. The fixed point $J_{*}^{\text {av }}$ remains unchanged as compared to Eq. (21) but the time constant $\tau^{\text {av }}$ for an approach of $J_{*}^{\text {av }}$ from below is increased by a factor (1 $\left.-N_{\uparrow} / N\right)^{-1} \geqslant 1$ as compared to Eq. (22). Similarly, $\tau^{\text {av }}$ for an approach of $J_{*}^{\text {av }}$ from above is increased by a factor ( 1 $\left.-N_{\downarrow} / N\right)^{-1} \geqslant 1$.

The factor by which $\tau^{\mathrm{av}}$ is increased is of order 1 , if we use the upper bound $\vartheta=(1+d) J_{*}^{\mathrm{av}}$, where $d>$ is of order 1 . If $J^{\text {av }}=J_{*}^{\text {av }}$, at most $N_{\uparrow}=N /(1+d)$ synapses can saturate at the upper bound comprising the total weight. The remaining $N_{\downarrow}=N-N /(1+d)$ synapses are at the lower bound 0 . The time constant $\tau^{\mathrm{av}}$ is enhanced by at most $1+1 / d$ and $1+d$ for an approach of the fixed point from below and above, respectively.

\section{APPENDIX E: RANDOM WALK OF SYNAPTIC WEIGHTS}

We consider the random walk of a synaptic weight $J_{i}(t)$ for $t>t_{0}$, where $J_{i}\left(t_{0}\right)$ is some starting value. The time course of $J_{i}(t)$ follows from Eq. (1),

$$
\begin{aligned}
J_{i}(t)=J_{i}\left(t_{0}\right) & +\int_{t_{0}}^{t} d t^{\prime}\left[w^{\text {in }} S_{i}^{\text {in }}\left(t^{\prime}\right)+w^{\text {out }} S^{\text {out }}\left(t^{\prime}\right)\right] \\
& +\int_{t_{0}}^{t} d t^{\prime} \int_{t_{0}}^{t} d t^{\prime \prime} W\left(t^{\prime \prime}-t^{\prime}\right) S_{i}^{\text {in }}\left(t^{\prime \prime}\right) S^{\text {out }}\left(t^{\prime}\right) .
\end{aligned}
$$

For a specific $i$, the spike trains $S_{i}^{\text {in }}\left(t^{\prime}\right)$ and $S^{\text {out }}\left(t^{\prime}\right)$ are now assumed to be statistically independent and generated by Poisson processes with constant rates $\nu^{\text {in }}$ for all $i$ and $\nu^{\text {out }}$, respectively; cf. Secs. II A and IV A. Here $\nu^{\text {in }}$ can be prescribed, whereas $\nu^{\text {out }}$ then follows; cf., for instance, Eq. (7). For large $N$ the independence is an excellent approximation. The learning parameters $w^{\text {in }}$ and $w^{\text {out }}$ can be positive or negative. The learning window $W$ is some quadratically integrable function with a width $\mathcal{W}$ as defined in Sec. II C. Finally, it may be beneficial to realize that spikes are described by $\delta$ functions.

The weight $J_{i}(t)$ is a stepwise constant function of time; see Fig. 2 (bottom). According to Eq. (E1), an input spike arriving at synapse $i$ at time $t$ changes $J_{i}$ at that time by a constant amount $w^{\text {in }}$ and a variable amount $\int_{t_{0}}^{t} d t^{\prime} W(t$ $\left.-t^{\prime}\right) S^{\text {out }}\left(t^{\prime}\right)$, which depends on the sequence of output spikes in the interval $\left[t_{0}, t\right]$. Similarly, an output spike at time $t$ results in a constant weight change $w^{\text {out }}$ and a variable one that equals $\int_{t_{0}}^{t} d t^{\prime \prime} W\left(t^{\prime \prime}-t\right) S_{i}^{\text {in }}\left(t^{\prime \prime}\right)$. We obtain a random walk with independent steps but randomly variable step size. Suitable rescaling of this random walk leads to Brownian motion.

As in Sec. II B, we substitute $s=t^{\prime \prime}-t^{\prime}$ in the second line of Eq. (E1) and extend the integration over the new variable $s$ so as to run from $-\infty$ to $\infty$. This does not introduce a big error for $t-t_{0} \gg \mathcal{W}$. The second line of Eq. (E1) then reduces to $\int d s W(s) \int_{t_{0}}^{t} d t^{\prime} S_{i}^{\text {in }}\left(t^{\prime}+s\right) S^{\text {out }}\left(t^{\prime}\right)$.

We denote ensemble averages by angular brackets \langle\rangle . The variance then reads

$$
\operatorname{var} J_{i}(t)=\left\langle J_{i}^{2}\right\rangle(t)-\left\langle J_{i}\right\rangle^{2}(t) .
$$

To simplify the ensuing argument, upper and lower bounds for each weight are not taken into account.

For the calculation of the variance in Eq. (E2), first of all we consider the term $\left\langle J_{i}\right\rangle(t)$. We use the notation $\left\langle S_{i}^{\text {in }}\right\rangle(t)$ $=\nu^{\text {in }}$ and $\left\langle S^{\text {out }}\right\rangle(t)=\nu^{\text {out }}$ because of constant input and output intensities. Stochastic independence of input and output leads to $\left\langle S_{i}^{\text {in }}\left(t^{\prime}+s\right) S^{\text {out }}\left(t^{\prime}\right)\right\rangle=\nu^{\text {in }} \nu^{\text {out }}$. Using Eq. (E1) and $\int d s W(s)=\widetilde{W}(0)$ we then obtain

$$
\left\langle J_{i}\right\rangle(t)=J_{i}\left(t_{0}\right)+\left(t-t_{0}\right)\left[w^{\text {in }} \nu^{\text {in }}+w^{\text {out }} \nu^{\text {out }}+\nu^{\text {in }} \nu^{\text {out }} \widetilde{W}(0)\right] .
$$

Next, we consider the term $\left\langle J_{i}^{2}\right\rangle(t)$ in Eq. (E2). Using Eq. (E1) once again we obtain for $t-t_{0} \gg \mathcal{W}$ 


$$
\begin{aligned}
\left\langle J_{i}^{2}\right\rangle(t)= & -J_{i}\left(t_{0}\right)^{2}+2 J_{i}\left(t_{0}\right)\left\langle J_{i}\right\rangle(t)+\int_{t_{0}}^{t} d t^{\prime} \int_{t_{0}}^{t} d u^{\prime} \\
& \times\left\{\left\langle S_{i}^{\text {in }}\left(t^{\prime}\right) S_{i}^{\text {in }}\left(u^{\prime}\right)\right\rangle\left(w^{\text {in }}\right)^{2}\right. \\
& +\left\langle S^{\text {out }}\left(t^{\prime}\right) S^{\text {out }}\left(u^{\prime}\right)\right\rangle\left(w^{\text {out }}\right)^{2} \\
& +\left\langle S_{i}^{\text {in }}\left(t^{\prime}\right) S^{\text {out }}\left(u^{\prime}\right)\right\rangle 2 w^{\text {in }} w^{\text {out }} \\
& +2 \int d s W(s)\left[\left\langle S_{i}^{\text {in }}\left(t^{\prime}\right) S_{i}^{\text {in }}\left(u^{\prime}+s\right) S^{\text {out }}\left(u^{\prime}\right)\right\rangle w^{\text {in }}\right. \\
& \left.+\left\langle S^{\text {out }}\left(t^{\prime}\right) S_{i}^{\text {in }}\left(u^{\prime}+s\right) S^{\text {out }}\left(u^{\prime}\right)\right\rangle w^{\text {out }}\right] \\
& +\int d s \int d v W(s) W(v) \\
& \left.\times\left\langle S_{i}^{\text {in }}\left(t^{\prime}+s\right) S_{i}^{\text {in }}\left(u^{\prime}+v\right) S^{\text {out }}\left(t^{\prime}\right) S^{\text {out }}\left(u^{\prime}\right)\right\rangle\right\} .
\end{aligned}
$$

Since input and output were assumed to be independent, we get

$$
\begin{aligned}
\left\langle S_{i}^{\text {in }} S_{i}^{\text {in }} S^{\text {out }} S^{\text {out }}\right\rangle & =\left\langle S_{i}^{\text {in }} S_{i}^{\text {in }}\right\rangle\left\langle S^{\text {out }} S^{\text {out }}\right\rangle, \\
\left\langle S_{i}^{\text {in }} S_{i}^{\text {in }} S^{\text {out }}\right\rangle & =\left\langle S_{i}^{\text {in }} S_{i}^{\text {in }}\right\rangle\left\langle S^{\text {out }}\right\rangle, \\
\left\langle S^{\text {out }} S^{\text {out }} S_{i}^{\text {in }}\right\rangle & =\left\langle S^{\text {out }} S^{\text {out }}\right\rangle\left\langle S_{i}^{\text {in }}\right\rangle .
\end{aligned}
$$

We note that $\left\langle S_{i}^{\text {in }}\right\rangle=\nu^{\text {in }}$ for all $i$ and $\left\langle S^{\text {out }}\right\rangle=\nu^{\text {out }}$.

Input spikes at times $t^{\prime}$ and $u^{\prime}$ are independent as long as $t^{\prime} \neq u^{\prime}$. In this case we therefore have $\left\langle S_{i}^{\text {in }}\left(t^{\prime}\right) S_{i}^{\text {in }}\left(u^{\prime}\right)\right\rangle$ $=\nu^{\text {in }} \nu^{\text {in }}$. For arbitrary times $t^{\prime}$ and $u^{\prime}$ we find (cf. Appendix A)

$$
\left\langle S_{i}^{\text {in }}\left(t^{\prime}\right) S_{i}^{\text {in }}\left(u^{\prime}\right)\right\rangle=\nu^{\text {in }}\left[\nu^{\text {in }}+\delta\left(t^{\prime}-u^{\prime}\right)\right] .
$$

Similarly, for the correlation between output spike trains we obtain

$$
\left\langle S^{\text {out }}\left(t^{\prime}\right) S^{\text {out }}\left(u^{\prime}\right)\right\rangle=\nu^{\text {out }}\left[\nu^{\text {out }}+\delta\left(t^{\prime}-u^{\prime}\right)\right] .
$$

Using Eqs. (E5), (E6), and (E7) in Eq. (E4), performing the integrations, and inserting the outcome together with Eq. (E3) into Eq. (E2), we arrive at

$$
\operatorname{var} J_{i}(t)=\left(t-t_{0}\right) D
$$

where

$$
\begin{aligned}
D= & \nu^{\text {in }}\left(w^{\text {in }}\right)^{2}+\nu^{\text {out }}\left(w^{\text {out }}\right)^{2}+\nu^{\text {in }} \nu^{\text {out }} \int d s W(s)^{2} \\
& +\nu^{\text {in }} \nu^{\text {out }} \widetilde{W}(0)\left[2\left(w^{\text {in }}+w^{\text {out }}\right)+\widetilde{W}(0)\left(\nu^{\text {in }}+\nu^{\text {out }}\right)\right]
\end{aligned}
$$

as announced.
[1] D.O. Hebb, The Organization of Behavior (Wiley, New York, 1949).

[2] C. von der Malsburg, Kybernetik 14, 85 (1973).

[3] R. Linsker, Proc. Natl. Acad. Sci. USA 83, 7508 (1986).

[4] R. Linsker, Proc. Natl. Acad. Sci. USA 83, 8390 (1986).

[5] D.J.C. MacKay and K.D. Miller, Network 1, 257 (1990).

[6] K.D. Miller, Neural Comput. 2, 321 (1990).

[7] K.D. Miller and D.J.C. MacKay, Neural Comput. 6, 100 (1994).

[8] S. Wimbauer, O.G. Wenisch, K.D. Miller, and J.L. van Hemmen, Biol. Cybern. 77, 453 (1997).

[9] S. Wimbauer, O.G. Wenisch, J.L. van Hemmen, and K.D. Miller, Biol. Cybern. 77, 463 (1997).

[10] D.J. Willshaw and C. von der Malsburg, Proc. R. Soc. London, Ser. B 194, 431 (1976).

[11] K. Obermayer, G.G. Blasdel, and K. Schulten, Phys. Rev. A 45, 7568 (1992).

[12] T. Kohonen, Self-Organization and Associative Memory (Springer, Berlin, 1984).

[13] L.M. Optican and B.J. Richmond, J. Neurophysiol. 57, 162 (1987).

[14] R. Eckhorn et al., Biol. Cybern. 60, 121 (1988).

[15] C.M. Gray and W. Singer, Proc. Natl. Acad. Sci. USA 86, 1698 (1989).

[16] C.E. Carr and M. Konishi, J. Neurosci. 10, 3227 (1990).

[17] W. Bialek, F. Rieke, R.R. de Ruyter van Steveninck, and D. Warland, Science 252, 1855 (1991).
[18] A.K. Kreiter and W. Singer, Eur. J. Neurosci. 4, 369 (1992).

[19] M. Abeles, in Models of Neural Networks II, edited by E. Domany, J.L. van Hemmen, and K. Schulten (Springer, New York, 1994), pp. 121-140.

[20] W. Singer, in Models of Neural Networks II, edited by E. Domany, J.L. van Hemmen, and K. Schulten (Springer, New York, 1994), pp. 141-173.

[21] J.J. Hopfield, Nature (London) 376, 33 (1995).

[22] W. Gerstner, R. Kempter, J.L. van Hemmen, and H. Wagner, Nature (London) 383, 76 (1996).

[23] R.C. deCharms and M.M. Merzenich, Nature (London) 381, 610 (1996).

[24] M. Meister, Proc. Natl. Acad. Sci. USA 93, 609 (1996).

[25] H. Markram, J. Lübke, M. Frotscher, and B. Sakmann, Science 275, 213 (1997).

[26] L.I. Zhang et al., Nature (London) 395, 37 (1998).

[27] B. Gustafsson et al., J. Neurosci. 7, 774 (1987).

[28] T.V.P. Bliss and G.L. Collingridge, Nature (London) 361, 31 (1993).

[29] D. Debanne, B.H. Gähwiler, and S.M. Thompson, Proc. Natl. Acad. Sci. USA 91, 1148 (1994).

[30] T.H. Brown and S. Chattarji, in Models of Neural Networks II, edited by E. Domany, J.L. van Hemmen, and K. Schulten (Springer, New York, 1994), pp. 287-314.

[31] C.C. Bell, V.Z. Han, Y. Sugawara, and K. Grant, Nature (London) 387, 278 (1997).

[32] V. Lev-Ram et al., Neuron 18, 1025 (1997). 
[33] H.J. Koester and B. Sakmann, Proc. Natl. Acad. Sci. USA 95, 9596 (1998).

[34] L.F. Abbott and K.I. Blum, Cereb. Cortex 6, 406 (1996).

[35] A.V.M. Herz, B. Sulzer, R. Kühn, and J.L. van Hemmen, Europhys. Lett. 7, 663 (1988).

[36] A.V.M. Herz, B. Sulzer, R. Kühn, and J.L. van Hemmen, Biol. Cybern. 60, 457 (1989).

[37] J.L. van Hemmen et al., in Konnektionismus in Artificial Intelligence und Kognitionsforschung, edited by G. Dorffner (Springer, Berlin, 1990), pp. 153-162.

[38] W. Gerstner, R. Ritz, and J.L. van Hemmen, Biol. Cybern. 69, 503 (1993).

[39] R. Kempter, W. Gerstner, J.L. van Hemmen, and H. Wagner, in Advances in Neural Information Processing Systems 8, edited by D. S. Touretzky, M. C. Mozer, and M. E. Hasselmo (MIT Press, Cambridge, MA, 1996), pp. 124-130.

[40] P. Häfliger, M. Mahowald, and L. Watts, in Advances in Neural Information Processing Systems 9, edited by M. C. Mozer, M. I. Jordan, and T. Petsche (MIT Press, Cambridge, MA, 1997), pp. 692-698.

[41] B. Ruf and M. Schmitt, in Proceedings of the 7th International Conference on Artificial Neural Networks (ICANN'97), edited by W. Gerstner, A. Germond, M. Hasler, and J.-D. Nicoud (Springer, Heidelberg, 1997), pp. 361-366.

[42] W. Senn, M. Tsodyks, and H. Markram, in Proceedings of the 7th International Conference on Artificial Neural Networks (ICANN'97), edited by W. Gerstner, A. Germond, M. Hasler, and J.-D. Nicoud (Springer, Heidelberg, 1997), pp. 121-126.

[43] R. Kempter, W. Gerstner, and J.L. van Hemmen, in Advances in Neural Information Processing Systems 11, edited by M. Kearns, S. A. Solla, and D. Cohn (MIT Press, Cambridge, MA, in press).

[44] J. W. Lamperti, Probability, 2nd ed. (Wiley, New York, 1996).

[45] J.A. Sanders and F. Verhulst, Averaging Methods in Nonlinear Dynamical Systems (Springer, New York, 1985).

[46] R. Kempter, W. Gerstner, J.L. van Hemmen, and H. Wagner, Neural Comput. 10, 1987 (1998).

[47] T.J. Sejnowski and G. Tesauro, in Neural Models of Plasticity: Experimental and Theoretical Approaches, edited by J. H. Byrne and W.O. Berry (Academic Press, San Diego, 1989), Chap. 6, pp. 94-103.

[48] R. Ritz and T.J. Sejnowski, Curr. Opin. Neurobiol. 7, 536 (1997).
[49] M. Meister, L. Lagnado, and D.A. Baylor, Science 270, 1207 (1995).

[50] M.J. Berry, D.K. Warland, and M. Meister, Proc. Natl. Acad. Sci. USA 94, 5411 (1997).

[51] W.E. Sullivan and M. Konishi, J. Neurosci. 4, 1787 (1984).

[52] C.E. Carr, Annu. Rev. Neurosci. 16, 223 (1993).

[53] C. Köppl, J. Neurosci. 17, 3312 (1997).

[54] R. Kempter, Hebbsches Lernen zeitlicher Codierung: Theorie der Schallortung im Hörsystem der Schleiereule, Naturwissenschaftliche Reihe, Vol. 17 (DDD, Darmstadt, 1997).

[55] L. Wiskott and T. Sejnowski, Neural Comput. 10, 671 (1998).

[56] P.A. Salin, R.C. Malenka, and R.A. Nicoll, Neuron 16, 797 (1996).

[57] W. Gerstner, R. Kempter, J.L. van Hemmen, and H. Wagner, in Pulsed Neural Nets, edited by W. Maass and C.M. Bishop (MIT Press, Cambridge, MA, 1998), Chap. 14, pp. 353-377.

[58] A. Zador, C. Koch, and T.H. Brown, Proc. Natl. Acad. Sci. USA 87, 6718 (1990).

[59] C.W. Eurich, J.D. Cowan, and J.G. Milton, in Proceedings of the 7th International Conference on Artificial Neural Networks (ICANN'97), edited by W. Gerstner, A. Germond, M. Hasler, and J.-D. Nicoud (Springer, Heidelberg, 1997), pp. 157-162.

[60] C.W. Eurich, U. Ernst, and K. Pawelzik, in Proceedings of the 8th International Conference on Artificial Neural Networks (ICANN'98), edited by L. Niklasson, M. Boden, and T. Ziemke (Springer, Berlin, 1998), pp. 355-360.

[61] H. Hüning, H. Glünder, and G. Palm, Neural Comput. 10, 555 (1998).

[62] N.G. van Kampen, Stochastic Processes in Physics and Chemistry (North-Holland, Amsterdam, 1992).

[63] H. Ritter, T. Martinez, and K. Schulten, Neural Computation and Self-Organizing Maps: An Introduction (Addison-Wesley, Reading, 1992).

[64] C. van Vreeswijk and H. Sompolinsky, Science 274, 1724 (1996).

[65] W. Softky and C. Koch, J. Neurosci. 13, 334 (1993).

[66] F. Rieke, D. Warland, R. de Ruyter van Steveninck, and W. Bialek, Spikes: Exploring the Neural Code (MIT Press, Cambridge, MA, 1997).

[67] M.N. Shadlen and W.T. Newsome, Curr. Opin. Neurobiol. 4, 569 (1994).

[68] Ö. Bernander, R.J. Douglas, K.A.C. Martin, and C. Koch, Proc. Natl. Acad. Sci. USA 88, 11569 (1991).

[69] M. Rapp, Y. Yarom, and I. Segev, Neural Comput. 4, 518 (1992). 